\title{
The Structure of Strategy-Proof Random Social Choice Functions over Product Domains and Lexicographically Separable PREFERENCES *
}

\author{
Shurojit Chatterji ${ }^{\dagger}$ Souvik Roy ${ }^{\ddagger}$ and Arunava Sen ${ }^{\S}$
}

June 21, 2012

\begin{abstract}
We characterize the class of dominant-strategy incentive-compatible (or strategyproof) random social choice functions in the standard multi-dimensional voting model where voter preferences over the various dimensions (or components) are lexicographically separable. We show that these social choice functions (which we call generalized random dictatorships) are induced by probability distributions on voter sequences of length equal to the number of components. They induce a fixed probability distribution on the product set of voter peaks. The marginal probability distribution over every component is a random dictatorship. Our results generalize the classic random dictatorship result in Gibbard (1977) and the decomposability results for strategyproof deterministic social choice functions for multi-dimensional models with separable preferences obtained in LeBreton and Sen (1999).
\end{abstract}

\section{INTRODUCTION}

Randomization has been used as a method of resolving conflicts of interest since antiquity. It has been analyzed extensively in a variety of models such as the pure voting model,

*We are particularly grateful to Huaxia Zeng for a careful reading of the paper and numerous helpful suggestions. We also thank a referee and the Editor for valuable comments. This research is supported by SMU Research Project 08-C244-SMU-014.

†Singapore Management University, Singapore.

${ }_{\ddagger}^{\ddagger}$ Indian Statistical Institute, New Delhi, India.

$\S$ Indian Statistical Institute, New Delhi, India. 
matching and auctions from the perspectives of aggregation, fairness and mechanism design. ${ }^{1}$ In the context of mechanism design, allowing for randomization expands the set of incentive-compatible social choice functions relative to the deterministic case. This is so because domain restrictions are inherent in the preference ranking of lotteries that satisfy the expected utility hypothesis. A classical result in this area is that of Gibbard (1977) which characterizes the class of strategy-proof random social choice functions over the complete domain of preferences.

In this paper we investigate the class of strategy-proof random social choice rules over a specific sub-domain of multi-dimensional (or multi-component) domains with separable preferences. The multi-dimensional separable preferences model is an important one with several applications and has been extensively studied in the deterministic setting, for example in Barberà et al. (1991), Barberà et al. (1993), LeBreton and Sen (1999), Barberà et al. (1997), Barberà et al. (2005) and Svensson and Torstensson (2008). For a survey see Sprumont (1995).

LeBreton and Sen (1999) show that every strategy-proof deterministic social choice function satisfying unanimity defined over a rich domain of preferences is decomposable, i.e. a strategy-proof social choice function is composed of strategy-proof social choice functions defined over each component domain. An immediate consequence of this result and the Gibbard-Satterthwaite Theorem (Gibbard (1973), Satterthwaite (1975)) is the following: if the domain is rich and each component domain is complete and has at least three alternatives, then a social choice function is strategy-proof if and only if there is a dictator for each component.

In this paper, we analyze the structure of random strategy-proof social choice functions (satisfying unanimity) defined over a domain where preferences are separable and components are lexicographically ordered. This is the domain of lexicographically separable preferences and is a subset of the domain of all separable preferences. This domain is rich in the sense of LeBreton and Sen (1999) (see their Example 3.3); according to the result referred to earlier, every deterministic strategy-proof social choice function over this domain must be a component random dictatorship. If the decomposability property extended straightforwardly to random social choice functions, we would expect strategy-proof random social choice functions (satisfying unanimity) over this domain to be the stochastic product of component random dictatorships. This is false; for instance, random dictatorship itself is clearly strategy-proof but not the product of component random dictatorships. The latter would put non-zero probabilities on alternatives that are not first-ranked by any voter unlike a random dictatorship. Thus products of component random dictatorships are strategy-proof but do not describe all random strategy-proof choice functions.

\footnotetext{
${ }^{1}$ See for example, Barberà and Sonnenschein (1978), Myerson (1981), Bogomolnaia and Moulin (2001), Bogomolnaia and Moulin (2004), Bogomolnaia et al. (2005), Moulin and Stong (2002).
} 
Our main result is a complete characterization of random strategy-proof social choice functions satisfying unanimity defined over lexicographically separable domains. We call such random social choice functions generalized random dictatorships and they include random dictatorships and products of component random dictatorships. A random dictatorship is a fixed probability distribution on the set of voters. At any preference profile, the probability of an alternative is the sum of the probability weights of voters for whom the alternative is the best. A generalized random dictatorship on the other hand, is a fixed probability distribution on the set of all voter sequences of length $m$ where $m$ is the number of components. For instance, if there are three voters and five components, there are $3^{5}$ possible voter sequences. A generalized random dictatorship assigns a probability to each of these sequences. An alternative is consistent with a sequence at a profile if each component of the alternative is the best (amongst all component alternatives) for the voter specified in the sequence for that component. The total probability of the alternative at the profile is simply the sum of probabilities of voter sequences consistent with the alternative. A generalized random dictatorship thus induces a fixed probability distribution on the product set of the maximal alternatives of all voters. A critical feature of these social choice functions is that the induced marginal probability distribution on each component is a random dictatorship.

The paper is organized as follows. In the next section, we introduce the model, the notation and the background results. The following section contains the main result and its proof while the final section concludes.

\section{BaCKGround AND PRELIMINARIES}

The set of alternatives is a finite set $A \equiv A_{1} \times A_{2} \ldots \times A_{m}$ where $A_{j}, j=1, \ldots m$ is the $j^{\text {th }}$ component set. The set of components will be written as $M=\{1, \ldots, m\}$. An element $a \in A$ is an $m$-tuple $a \equiv\left(a_{1}, \ldots, a_{m}\right)$. For any $Q \subset M$, we will let $A_{Q}=\prod_{j \in Q} A_{j}$. Abusing notation

slightly, we will write $A_{M-j}$ for the set $\prod_{k \neq j} A_{k}$. Typical elements of $A_{M-j}$ will be denoted by $a_{M-j}, b_{M-j}$ etc.

\subsection{PREFERENCES}

The set of voters is $I=\{1, . ., N\}$. Each voter $i$ has an antisymmetric preference ordering $P^{i}$ over the elements of $A$ which is assumed to be separable.

Definition 1 The ordering $P^{i}$ is separable if for all $Q \subset M$, for all $a_{Q}, b_{Q} \in A_{Q}$, for all $c_{M-Q}, d_{M-Q} \in A_{M-Q}$

$$
\left[\left(a_{Q}, c_{M-Q}\right) P^{i}\left(b_{Q}, c_{M-Q}\right)\right] \Rightarrow\left[\left(a_{Q}, d_{M-Q}\right) P^{i}\left(b_{Q}, d_{M-Q}\right)\right]
$$


If a preference ordering is separable, then choices over a subset of components do not affect ranking of alternatives over the remaining components. In other words, choices over components do not impose "externalities" over other components.

A particular class of separable orderings is the class of lexicographically separable or simply lexicographic orderings.

Definition 2 The ordering $P^{i}$ is lexicographic if there exists an antisymmetric ordering $\succ$ on the set $M$ and antisymmetric orderings $P_{j}^{i}$ on each component set $A_{j}, j \in M$ such that, for all $a, b \in A, a P^{i} b$ iff there exists a component $j$ such that

1. $a_{j} P_{j}^{i} b_{j}$

2. $a_{k}=b_{k}$ for all $k \in M$ such that $k \succ j$.

Let $P^{i}$ be a lexicographic ordering. We shall refer to the components that are maximal and minimal according to the ordering $\succ$ over $M$ as the lexicographically best and lexicographically worst components respectively. In general if, components $j$ and $k$ are such that $k \succ j$, we shall say that component $k$ is lexicographically better than component $j$.

Let $\mathbb{P}, \mathbb{D}^{S}$ and $\mathbb{D}^{L}$ denote respectively the set of all antisymmetric orderings, the set of separable orderings, the set of additively separable orderings and the set of lexicographic preference orderings over $A$ respectively. We note that $\mathbb{D}^{L} \subseteq \mathbb{D}^{S} \subset \mathbb{P} .{ }^{2}$

A separable preference $P^{i}$ induces a marginal preference ordering $P_{Q}^{i}$ over $A_{Q}, Q \subset M$ in a natural way: for every $a_{Q}, b_{Q} \in A_{Q}$

$$
\left[a_{Q} P_{Q}^{i} b_{Q}\right] \text { if }\left[\left(a_{Q}, c_{M-Q}\right) P^{i}\left(b_{Q}, c_{M-Q}\right) \text { for all } c_{M-Q} \in A_{M-Q}\right] .
$$

Let $\mathbb{D} \subset \mathbb{D}^{S}$. The set of marginal preference orderings over components in the set $Q \subset M$ will be denoted by $\mathbb{D}_{Q}$. If $Q$ consists of a single component, we shall write $\mathbb{D}_{j}$ for the set of orderings induced by $\mathbb{D}$ over $A_{j}$.

For any $\mathbb{D} \subset \mathbb{P}$, a preference profile $P$ is an $N$-tuple $\left(P^{1}, \ldots, P^{N}\right) \in \mathbb{D}^{N}$. For any voter $i$, ordering $\bar{P}^{i}$ and profile $P$, we shall let $\left(\bar{P}^{i}, P^{-i}\right)$ denote the profile where the $i^{\text {th }}$ component of $P$ has been replaced by $\bar{P}^{i}$. A marginal preference profile for components $Q$ is similarly an $N$-tuple, $P_{Q} \equiv\left(P_{Q}^{1}, \ldots, P_{Q}^{N}\right) \in \mathbb{D}^{N}$ where $\mathbb{D} \subset \mathbb{D}^{S}$. We shall say that two profiles $P, \bar{P} \in \mathbb{D}^{N}$ $\left(\mathbb{D} \subset \mathbb{D}^{S}\right)$ are marginally equivalent if $P_{j}^{i}=\bar{P}_{j}^{i}$ for all voters $i \in I$ and $j \in M$.

\footnotetext{
${ }^{2}$ The set of lexicographic orderings coincides with the separable orderings in the special case when there are two components and exactly two alternatives in every component set. In general $\mathbb{D}^{L}$ is a strict subset of $\mathbb{D}^{S}$.
} 


\subsection{Random Social Choice Functions}

We let $\mathcal{L}(A)$ denote the set of lotteries over the elements of the set $A$. If $\lambda \in \mathcal{L}(A)$, then $\lambda_{a}$ will denote the probability that $\lambda$ puts on $a \in A$. Clearly $\lambda_{a} \geq 0$ and $\sum_{a \in A} \lambda_{a}=1$. For every $j \in M$ we can define $\mathcal{L}\left(A_{j}\right)$ accordingly.

Definition 3 Let $\mathbb{D} \subset \mathbb{P}$. A Random Social Choice Function (RSCF) (for the domain $\mathbb{D}$ ) is a $\operatorname{map} \varphi: \mathbb{D}^{N} \rightarrow \mathcal{L}(A)$.

Our focus is on RSCFs that are strategy-proof, i.e. those that provide voters with dominant-strategy incentives to reveal their preference orderings (which are assumed to be private information), truthfully. In models such as ours where the outcome of voting is a probability distribution over outcomes, there are several ways to define strategy-proofness. Here we follow the approach of Gibbard (1977).

Definition $4 A$ utility function $u: A \rightarrow \Re$ represents the ordering $P^{i}$ over $A$ if for all $a, b \in A$,

$$
\left[a P^{i} b\right] \Leftrightarrow[u(a)>u(b)]
$$

Definition $5 A R S C F \varphi: \mathbb{D}^{N} \rightarrow \mathcal{L}(A)$ is manipulable by voter $i$ at profile $P \in \mathbb{D}^{N}$ via $\bar{P}^{i} \in \mathbb{D}$ if there exists a utility functions $u$ representing $P^{i}$ such that

$$
\sum_{a \in A} u(a) \varphi_{a}\left(\bar{P}^{i}, P^{-i}\right)>\sum_{a \in A} u(a) \varphi_{a}\left(P^{i}, P^{-i}\right) .
$$

Definition 6 A RSCF $\varphi: \mathbb{D}^{N} \rightarrow \mathcal{L}(A)$ is strategy-proof if it is not manipulable by any voter at any profile. Equivalently, $\varphi$ is strategy-proof if, for all $i \in I$, for all $P \in \mathbb{D}^{N}$, for all $\bar{P}^{i} \in \mathbb{D}$ and all utility functions $u$ representing $P^{i}$, we have

$$
\sum_{a \in A} u(a) \varphi_{a}\left(P^{i}, P^{-i}\right) \geq \sum_{a \in A} u(a) \varphi_{a}\left(\bar{P}^{i}, P^{-i}\right) .
$$

A RSCF is strategy-proof if at every profile no voter can obtain a higher expected utility by deviating from her true preference ordering than she would if she announced her true preference ordering. Here, expected utility is computed with respect an arbitrary utility representation of her true preferences. It is well-known that this is equivalent to requiring that the probability distribution from truth-telling stochastically dominates the probability distribution from misrepresentation in terms of a voter's true preferences. This is stated formally below.

For any $i \in I, P^{i} \in \mathbb{D}$ and $a \in A$, we let $B\left(a, P^{i}\right)=\left\{b \in A: b P^{i} a\right\} \cup\{a\}$, i.e. $B\left(a, P^{i}\right)$ denotes the set of alternatives that are weakly preferred to $a$ according to the ordering $P^{i}$.

Definition $7 A R S C F \varphi: \mathbb{D}^{N} \rightarrow \mathcal{L}(A)$ is manipulable by voter $i$ at profile $P \in \mathbb{D}^{N}$ via $\bar{P}^{i} \in \mathbb{D}$ if there exists $a \in A$ such that 


$$
\sum_{b \in B\left(a, P^{i}\right)} \varphi_{b}\left(\bar{P}^{i}, P^{-i}\right)>\sum_{b \in B\left(a, P^{i}\right)} \varphi_{b}\left(P^{i}, P^{-i}\right) .
$$

It is strategy-proof if for all $i \in I$, for all $P \in \mathbb{D}^{N}$, for all $\bar{P}_{i} \in \mathbb{D}$ and all $a \in A$, we have

$$
\sum_{b \in B\left(a, P^{i}\right)} \varphi_{b}\left(P^{i}, P^{-i}\right) \geq \sum_{b \in B\left(a, P^{i}\right)} \varphi_{b}\left(\bar{P}^{i}, P^{-i}\right) .
$$

We also assume throughout the paper that RSCFs satisfy the standard (and mild) requirement of unanimity. This requires an alternative that is first-ranked by all voters in any profile to be selected with probability one in that profile. For any $P^{i} \in \mathbb{D}$, let $\tau\left(P^{i}, A\right)$ denote the maximal element in $A$ according to $P^{i}$. Since the domain consists of antisymmetric orderings and $A$ is finite, a maximal element always exists and is unique.

Definition 8 A RSCF $\varphi: \mathbb{D}^{N} \rightarrow \mathcal{L}(A)$ satisfies unanimity if for all $P \in \mathbb{D}^{N}$ and $a \in A$,

$$
\left[a=\tau\left(P^{i}, A\right) \text { for all } i \in I\right] \Rightarrow\left[\varphi_{a}(P)=1\right] .
$$

We now review some existing results that have a bearing on the results in this paper.

\subsection{Existing Results}

A RSCF of particular significance is random dictatorship.

Definition 9 Let $\mathbb{D} \subset \mathbb{P}$. The $R S C F \varphi^{r}: \mathbb{D}^{N} \rightarrow \mathcal{L}(A)$ is a random dictatorship if there exist non-negative real numbers $\beta^{i}, i \in I$ with $\sum_{i \in I} \beta^{i}=1$ such that for all $P \in \mathbb{D}^{N}$ and $a \in A$,

$$
\varphi_{a}^{r}(P)=\sum_{\left\{i: \tau\left(P^{i}, A\right)=a\right\}} \beta^{i}
$$

In a random dictatorship, each voter $i$ gets weight $\beta_{i}$ where the sum of these $\beta_{i}$ 's is one. At any profile, the probability assigned to an alternative $a$ is simply the sum of the weights of the voters whose maximal element is $a$. A random dictatorship is clearly strategy-proof for any domain; by misrepresentation, a voter can only transfer weight from her most-preferred to a less-preferred alternative. A fundamental result in Gibbard (1977) states that the converse is also true for the complete domain $\mathbb{P} .{ }^{3}$

Theorem 1 [Gibbard (1977) ] Assume $|A| \geq 3$. A RSCF $\varphi: \mathbb{P}^{N} \rightarrow \mathcal{L}(A)$ is strategy-proof and satisfies unanimity if and only if it is a random dictatorship.

\footnotetext{
${ }^{3}$ Gibbard's result is actually more general than Theorem 1 below because it does not assume unanimity. However since unanimity will be a maintained hypothesis throughout the paper, we state only the version of the result with unanimity. See also Sen (2011) for a generalization of the Gibbard result with unanimity.
} 
We now recall results for deterministic social choice functions. For convenience, we provide definitions only for domains $\mathbb{D} \subset \mathbb{D}^{S}$.

Definition 10 A deterministic social choice function (DSCF) $f$ is a map $f: \mathbb{D}^{N} \rightarrow A$.

A DSCF is simply a RSCF whose image set is the set of degenerate probability distributions over $A$. The definitions of strategy-proofness and unanimity for a DSCF are special cases of those of RSCFs and are omitted.

Definition 11 A DSCF $f:\left[\mathbb{D}^{L}\right]^{N} \rightarrow A$ is a component dictatorship if there exists a map $\sigma: M \rightarrow N$ such that for all $P \in\left[\mathbb{D}^{L}\right]^{N}$,

$$
[f(P)=a] \Rightarrow\left[a_{j}=\tau\left(P_{j}^{\sigma(j)}, A_{j}\right)\right]
$$

In a component dictatorship, the $j^{\text {th }}$ component of the outcome at a profile is the maximal element of the $j^{\text {th }}$ component of voter $\sigma(j)$. Since preferences are separable for all individuals, these maximal elements are well-defined. We can imagine the DSCF being decomposable into component DSCFs which are dictatorial.

Theorem 2 [LeBreton and Sen (1999)] Assume $\left|A_{j}\right| \geq 3$ for all $j \in M$. A DSCF $f$ : $\left[\mathbb{D}^{L}\right]^{N} \rightarrow A$ is strategy-proof and satisfies unanimity if and only if it is a component dictatorship.

The result is an immediate consequence of Theorem 5.1, in LeBreton and Sen (1999).

\subsection{Generalized Random Dictatorships}

Does the decomposability property carry over to RSCFs? A particular generalization of component dictatorship is a RSCF where the probability distribution over the set $A$ is the product of component random dictatorships. We define this below.

Definition 12 A RSCF is $\varphi:\left[\mathbb{D}^{L}\right]^{N} \rightarrow \mathcal{L}(A)$ is an independent component random dictatorship if for each $j \in M$, there exists a random dictatorship $\varphi_{j}: \mathbb{P}_{j}{ }^{N} \rightarrow \mathcal{L}\left(A_{j}\right)$ such that for all $P \in\left[\mathbb{D}^{L}\right]^{N}$

$$
\varphi(P)=\prod_{j \in M} \varphi_{j}\left(P_{j}\right)
$$

Consider the case where there are two voters and two components. Suppose the weight vectors for components 1 and 2 are $\left(\beta_{1}^{1}, \beta_{1}^{2}\right)$ and $\left(\beta_{2}^{1}, \beta_{2}^{2}\right)$ respectively. Then in any profile where voter 1 and 2's maximal elements are $a_{1} a_{2}$ and $b_{1} b_{2}$ respectively, the alternatives $a_{1} a_{2}, a_{1} b_{2}, b_{1} a_{2}$ and $b_{1} b_{2}$ get probability weights $\beta_{1}^{1} \beta_{2}^{1}, \beta_{1}^{1} \beta_{2}^{2}, \beta_{2}^{1} \beta_{1}^{2}$ and $\beta_{1}^{2} \beta_{2}^{2}$ respectively. 
An independent component random dictatorship is strategy-proof (we shall verify this later) and clearly satisfies unanimity. Is every strategy-proof RSCF defined over the domain $\mathbb{D}^{S}$ with $\left|A_{j}\right| \geq 3$ an independent component random dictatorship? No, and this is established by the observation that a random dictatorship is strategy-proof but not an independent component random dictatorship unless it is deterministic, i.e. there exists a voter $i$ such that $\beta^{i}=1$. Showing that a random dictatorship is strategy-proof is routine. To demonstrate the other claim, consider for simplicity the case where there are two voters $i$ and $k$ and two components. Suppose also that $\beta^{i}, \beta^{k}>0$. Consider a profile where $i$ 's maximal element is $a_{1} a_{2}$ and $k$ 's is $b_{1} b_{2}$ where $a_{1} \neq b_{1}$ and $a_{2} \neq b_{2}$. Observe that this RSCF would put probabilities $\beta^{i}$ and $\beta^{k}$ on $a_{1} a_{2}$ and $b_{1} b_{2}$ respectively and zero on all other alternatives. However every independent component random dictatorship which puts strictly positive probability on $a_{1} a_{2}$ and $b_{1} b_{2}$ also puts strictly positive probability on the alternatives $a_{1} b_{2}$ and $b_{1} a_{2}$.

Below, we formulate a generalization of both random dictatorship and independent component random dictatorship which coincides with the class of strategy-proof RSCFs satisfying unanimity in the case where each component set has at least three alternatives.

Let $\underline{i} \equiv\left(i_{1}, \ldots, i_{m}\right) \in I^{m}$ be an $m$-tuple of voters. We shall call such an $m$-tuple, a voter sequence. We note that a voter may appear multiple times in a voter sequence. For all $a \in A$ and $P \in\left[\mathbb{D}^{S}\right]^{N}$, we shall let $\chi(a, P)$ denote the set of voter sequences consistent with $a$ and $P$ where $\chi(a, P)=\left\{\underline{i} \in I^{m}: a_{j}=\tau\left(P_{j}^{i_{j}}, A_{j}\right) \quad\right.$ for all $\left.j=1, \ldots m\right\}$.

Definition 13 A RSCF $\varphi^{g}:\left[\mathbb{D}^{S}\right]^{N} \rightarrow \mathcal{L}(A)$ is a generalized random dictatorship if there exist non-negative real numbers $\gamma(\underline{i})$ for all $\underline{i} \in I^{m}$ with $\sum_{\underline{i} \in I^{m}} \gamma(\underline{i})=1$ such that for all $a \in A$ and $P \in\left[\mathbb{D}^{S}\right]^{N}$,

$$
\varphi_{a}^{g}(P)=\sum_{\underline{i} \in \chi(a, P)} \gamma(\underline{i}) .
$$

Consider the following example. Suppose $I=\{1,2\}$ and $A_{j}=\left\{a_{j}, b_{j}, c_{j}\right\}$ with $j=1,2$. Here $\underline{i}$ is one of four, two-tuples $(1,1),(1,2),(2,1)$ and $(2,2)$. The function $\gamma$ specifies four non-negative real numbers $\gamma(1,1), \gamma(1,2), \gamma(2,1)$ and $\gamma(2,2)$ which sum to one. Consider a profile $P$ where the maximal alternatives of voters 1 and 2 are $\left(a_{1} a_{2}\right)$ and $\left(b_{1} b_{2}\right)$ respectively. Observe that $\chi\left(\left(a_{1} a_{2}\right), P\right)=\{(1,1)\}, \chi\left(\left(a_{1} b_{2}\right), P\right)=\{(1,2)\}, \chi\left(\left(b_{1} a_{2}\right), P\right)=\{(2,1)\}$ and $\chi\left(\left(b_{1} b_{2}\right), P\right)=\{(2,2)\}$. Hence, a generalized random dictatorship puts probabilities of $\gamma(1,1), \gamma(1,2), \gamma(2,1)$ and $\gamma(2,2)$ on $\left(a_{1} a_{2}\right),\left(a_{1} b_{2}\right),\left(b_{1} a_{2}\right)$ and $\left(b_{1} b_{2}\right)$ respectively and zero on all other alternatives. Consider another profile $\bar{P}$ where voter 1 and 2's maximal alternatives are $\left(a_{1}, c_{2}\right)$ and $\left(a_{1} b_{2}\right)$ respectively. Here $\chi\left(\left(a_{1} c_{2}\right), \bar{P}\right)=\{(1,1),(2,1)\}$, and $\chi\left(\left(a_{1} b_{2}\right), P\right)=\{(1,2),(2,1)\}$. Hence this RSCF will put probability $\gamma(1,1)+\gamma(2,1)$ on $\left(a_{1} c_{2}\right)$ and $\gamma(1,2)+\gamma(2,2)$ on $\left(a_{1} b_{2}\right)$ and zero on everything else.

In general, a generalized random dictatorship is specified by $N^{m}$ non-negative real numbers adding up to one. For any $\underline{i} \equiv\left(i_{1}, \ldots, i_{m}\right)$, the probability of an alternative $a$ in profile 
$P$ is the sum of $\gamma(\underline{i})$ 's over those $\underline{i}$ 's which have the property that for every $j=1, \ldots, m, a_{j}$ is the maximal element in $A_{j}$ for voter $i_{j}$, i.e. over all elements of the set $\chi(a, P)$.

We make several observations about generalized random dictatorships.

OBSERVATION 1 The value of a generalized random dictatorship at a profile depends only on the maximal alternatives (or "tops") of voter preferences at the profile. However it may assign positive probabilities to all elements of the product set of the top alternatives. In other words, a generalized random dictatorship is a probability distribution over the set $\prod_{j \in M}\left\{\tau\left(P_{j}^{1}, A_{j}\right), \ldots, \tau\left(P_{j}^{N}, A_{j}\right)\right\}$.

OBSERVATION 2 Let $\varphi^{g}$ be a generalized random dictatorship with an associated map $\gamma$. Pick a component $j$, a voter $s$ and let $\beta^{s}=\sum_{\left\{\underline{i} \equiv\left(i_{1}, \ldots, i_{m}\right): i_{j}=s\right\}} \gamma(\underline{i})$. Clearly $0 \leq \beta^{s} \leq 1$ and $\sum_{s \in I} \beta^{s}=1$. For any profile $P \in\left[\mathbb{D}^{S}\right]^{N}$, observe that the probability of $a_{j} \in A_{j}$ in the marginal distribution $\varphi_{j}^{g}(P)$ is $\sum_{\left\{s: \tau\left(P_{j}^{s}\right)=a_{j}\right\}} \beta^{s}$. Hence, a generalized random dictatorship induces a marginal random social choice function over each component that is a random dictatorship with respect to marginal preferences over that component. More formally, if $\varphi^{g}$ is a generalized random dictatorship, there exist component random dictatorships $\varphi_{j}^{r}: \mathbb{P}^{N} \rightarrow$ $\mathcal{L}\left(A_{j}\right), j=1, \ldots, m$ such that for all $P \in\left[\mathbb{D}^{S}\right]^{N}, \varphi_{j}^{g}(P)=\varphi_{j}^{r}\left(P_{j}\right)$ for each $j=1, \ldots m$.

OBSERVATION 3 A random dictatorship is a special case of a generalized random dictatorship when $\gamma(\underline{i})=0$ for all voter sequences $\underline{i}$ such that $i_{j} \neq i_{j^{\prime}}$ for some $j \neq j^{\prime}$. Equivalently, $\gamma(\underline{i})>0$ implies $\underline{i}=(i, i, \ldots, i)$ for some $i \in I$.

OBSERVATION 4 An independent component random dictatorship is a special case of a generalized random dictatorship. Define the component random dictatorships as follows: for all $j=1, \ldots, m$, let $\gamma_{j}(i), i=1, \ldots, N$ be non-negative real numbers with $\sum_{i \in I} \gamma_{j}(i)=1$. Now define a generalized random dictatorship as follows: for all voter sequences $\underline{i} \equiv\left(i_{1}, \ldots, i_{m}\right)$, $\gamma(\underline{i})=\gamma_{1}\left(i_{1}\right) \times \gamma_{2}\left(i_{2}\right) \times \ldots \times \gamma_{m}\left(i_{m}\right)$.

ObSERVATION 5 In the special case where $m=1$, a generalized random dictatorship is simply a random dictatorship.

In the next section we show that all strategy-proof RSCFs satisfying unanimity are generalized random dictatorships.

\section{The Result}

Our main result is the following.

Theorem 3 Assume $\left|A_{j}\right| \geq 3$ for all $j=1, \ldots, m$. A RSCF $\varphi:\left[\mathbb{D}^{L}\right]^{N} \rightarrow \mathcal{L}(A)$ is strategyproof and satisfies unanimity if and only if it is a generalized random dictatorship. 
Proof: (Sufficiency) We show that generalized random dictatorships satisfy unanimity and strategy-proofness over the domain $\mathbb{D}^{S}$. Unanimity follows from Observation 1 . We show strategy-proofness. Let $\varphi^{g}$ be a generalized random dictatorship specified by the function $\gamma$ in Definition 13. Let $P \in\left[\mathbb{D}^{S}\right]^{N}$ be an arbitrary profile and let $i$ be an arbitrary voter. Consider a possible manipulation by $i$ at $P$ via $\bar{P}^{i}$. It follows from the definition of a generalized random dictatorship that the value of $\varphi^{g}$ at any profile depends only on the maximal alternatives of voters at the profile. Let $\tau\left(P^{i}\right)=a$ and $\tau\left(\bar{P}^{i}\right)=b$ where $b_{Q} \neq a_{Q}$ and $a_{M-Q}=b_{M-Q}$ for some non-empty subset $Q$ of $M$.

Pick an arbitrary $\underline{i} \equiv\left(i_{1}, \ldots, i_{m}\right) \in I^{m}$. If $i_{j} \neq i$ for any $j \in Q$ then probability $\gamma(\underline{i})$ is assigned to the same alternative under profiles $P$ and $\left(\bar{P}^{i}, P_{-i}\right)$. If $i_{j}=i$ for all $j \in T$ for some $T \subseteq Q$, then probability $\gamma(\underline{i})$ is shifted from alternative $\left(a_{T}, x_{M-T}\right)$ for some $x_{M-T} \in A_{M-T}$ in profile $P$ to $\left(b_{T}, x_{M-T}\right)$ in profile $\left(\bar{P}^{i}, P_{-i}\right)$. However $a_{j} P_{j}^{i} b_{j}$ for all $j \in T$ by assumption so that $\left(a_{T}, x_{M-T}\right) P^{i}\left(b_{T}, x_{M-T}\right)$ by separability. Therefore the distribution $\varphi^{g}\left(\bar{P}^{i}, P^{-i}\right)$ is obtained from $\varphi^{g}(P)$ by transferring probabilities from higher-ranked alternatives to lowerranked alternatives according to $P^{i}$. Clearly $\varphi^{g}(P)$ stochastically dominates $\varphi^{g}\left(\bar{P}^{i}, P^{-i}\right)$ according to $P^{i}$ and $\varphi^{g}$ is strategy-proof.

(Necessity) We proceed as follows. The proof consists of three steps. In Step 1, we establish an important "conditional unanimity" property; in Step 2 we establish generalized random dictatorship in the case of two voters and in Step 3 we extend the result to an arbitrary number of voters using induction on the number of voters.

We begin with a Lemma which holds for arbitrary domains and is a straightforward adaptation of a result in Gibbard (1977).

Let $\mathbb{D}$ be an arbitrary domain. Let $P^{i} \in \mathbb{D}$ and let $x, y \in A$ and assume that $x P^{i} y$. We say $x$ and $y$ are contiguous in $P^{i}$ if there does not exist $z \in A$ distinct from $x$ and $y$ such that $x P^{i} z P^{i} y$. We say that the ordering $\bar{P}_{i}$ is a feasible local switch of $x$ and $y$ in $P^{i}$ if (i) $x$ and $y$ are contiguous (ii) $x P^{i} y$ and $y \bar{P}^{i} x$ (iii) $B\left(x, P^{i}\right) \cup\{y\}=B\left(y, \bar{P}^{i}\right) \cup\{x\}$ (iv) $\bar{P}^{i} \in \mathbb{D}$.

Lemma 1 Let $\varphi: \mathbb{D}^{N} \rightarrow \mathcal{L}(A)$ be strategy-proof. Let $i$ be an arbitrary voter and let $\bar{P}^{i}$ be a feasible local switch of $x$ and $y$ in $P^{i}$. Then

(i) $\varphi_{y}\left(\bar{P}^{i}, P^{-i}\right) \geq \varphi_{y}(P)$.

(ii) $\varphi_{x}\left(\bar{P}^{i}, P^{-i}\right)+\varphi_{y}\left(\bar{P}^{i}, P^{-i}\right)=\varphi_{x}(P)+\varphi_{y}(P)$.

We omit the proof of this Lemma which is an implication of the definition of strategyproofness.

Consider an arbitrary strategy-proof $\operatorname{RSCF} \varphi:\left[\mathbb{D}^{L}\right]^{N} \rightarrow \mathcal{L}(A)$ satisfying unanimity. We will show that $\varphi$ is a generalized random dictatorship. Recall that every $P^{i} \in \mathbb{D}^{L}$ induces an ordering $P_{Q}^{i}$ over $A_{Q}$ for every $Q \subset M$. 
We establish some preliminary lemmas. Let $\succ$ be an ordering over the set $M$ and let $j \in M$. Then $E(\succ, j)=\{i \in M: i \succ j\}$. Thus $E(\succ, j)$ is the set of components which lexicographically dominate $j$.

Lemma 2 Let $P \in\left[\mathbb{D}^{L}\right]^{N}$ and $i \in I$. Let $P^{i}$ and $\bar{P}^{i}$ be lexicographic with respect to $\succ$ and $\succ^{\prime}$ and let $j$ be a component such that (i) $E(\succ, j)=E\left(\succ^{\prime}, j\right)=Q$ and (ii) $P_{Q \cup\{j\}}^{i}=\bar{P}_{Q \cup\{j\}}^{i}$. Then

(a) $\varphi_{k}(P)=\varphi_{k}\left(\bar{P}^{i}, P^{-i}\right)$ for all $k \in Q \cup\{j\}$.

(b) Let $a_{Q} \in A_{Q}$. Then $\varphi_{Q \cup\{j\}, a_{Q \cup\{j\}}}(P)=\varphi_{Q \cup\{j\}, a_{Q \cup\{j\}}}\left(\bar{P}^{i}, P^{-i}\right)$.

Before proving the Lemma, we provide an intermediate Proposition.

Proposition 1 For all $P^{i}, \bar{P}^{i} \in \mathbb{D}^{L}, P^{-i} \in\left[\mathbb{D}^{L}\right]^{N-1}$ and $T \subset M(T \neq \emptyset)$, if $\varphi_{T}\left(P^{i}, P^{-i}\right)=$ $\varphi_{T}\left(\bar{P}^{i}, P^{-i}\right)$, then $\varphi_{\hat{T}}\left(P^{i}, P^{-i}\right)=\varphi_{\hat{T}}\left(\bar{P}^{i}, P^{-i}\right)$ for all $\hat{T} \subset T$ where $\hat{T} \neq \emptyset$.

Proof: For all $a_{\hat{T}} \in A_{\hat{T}}$, we have the following:

$$
\begin{aligned}
\varphi_{\hat{T}, a_{\hat{T}}}\left(P^{i}, P^{-i}\right) & =\sum_{x_{M-\hat{T}} \in A_{M-\hat{T}}} \varphi_{\left(a_{\hat{T}}, x_{M-\hat{T}}\right)}\left(P^{i}, P^{-i}\right) \\
& =\sum_{x_{T-\hat{T}} \in A_{T-\hat{T}}} \sum_{x_{M-T} \in A_{M-T}} \varphi_{\left(a_{\hat{T}}, x_{T-\hat{T}}, x_{M-T}\right)}\left(P^{i}, P^{-i}\right) \\
& =\sum_{x_{T-\hat{T}} \in A_{T-\hat{T}}} \varphi_{T,\left(a_{\hat{T}}, x_{T-\hat{T}}\right)}\left(P^{i}, P^{-i}\right) \\
& =\sum_{x_{T-\hat{T}} \in A_{T-\hat{T}}} \varphi_{T,\left(a_{\hat{T}}, x_{T-\hat{T}}\right)}\left(\bar{P}^{i}, P^{-i}\right) \\
& =\sum_{x_{T-\hat{T}} \in A_{T-\hat{T}}} \sum_{x_{M-T} \in A_{M-T}} \varphi_{\left(a_{\hat{T}}, x_{T-\hat{T}}, x_{M-T}\right)}\left(\bar{P}^{i}, P^{-i}\right) \\
& =\sum_{x_{M-\hat{T}} \in A_{M-\hat{T}}} \varphi_{\left(a_{\hat{T}}, x_{M-\hat{T}}\right)}\left(\bar{P}^{i}, P^{-i}\right) \\
& =\varphi_{\hat{T}, a_{\hat{T}}}\left(\bar{P}^{i}, P^{-i}\right)
\end{aligned}
$$

We now return to the proof of the Lemma.

Proof: In view of Lemma 1, in order to show $(a)$, it suffices to show $(b)$.

To simplify the notation, let $\bar{Q}=Q \cup\{j\}$. Now, suppose $\varphi_{\bar{Q}}(P) \neq \varphi_{\bar{Q}}\left(\bar{P}^{i}, P^{-i}\right)$. Let $c_{\bar{Q}}$ be the $P_{\bar{Q}}^{i}-$ maximal alternative such that $\varphi_{\bar{Q}, c_{\bar{Q}}}\left(P^{i}, P^{-i}\right) \neq \varphi_{\bar{Q}, c_{\bar{Q}}}\left(\bar{P}^{i}, P^{-i}\right)$. Therefore, 
for all $x_{\bar{Q}} \in A_{\bar{Q}}$ with $x_{\bar{Q}} P_{\bar{Q}}^{i} c_{\bar{Q}}$, we have $\varphi_{\bar{Q}, x_{\bar{Q}}}(P)=\varphi_{\bar{Q}, x_{\bar{Q}}}\left(\bar{P}^{i}, P^{-i}\right)$. Then, we consider the following 2 cases.

Case 1: $\varphi_{\bar{Q}, c_{\bar{Q}}}(P)<\varphi_{\bar{Q}, c_{\bar{Q}}}\left(\bar{P}^{i}, P^{-i}\right)$.

Let $w_{M-\bar{Q}}$ denote the $P_{M-\bar{Q}}^{i}-$ minimal alternative. Consider $\left(c_{\bar{Q}}, w_{M-\bar{Q}}\right)$ and $\bar{B}=$ $B\left(\left(c_{\bar{Q}}, w_{M-\bar{Q}}\right), P^{i}\right)$. Since all components in $\bar{Q}$ lexicographically dominate all components in $M-\bar{Q}$, we know that $\bar{B}=\left\{x \in A\right.$ : either $x_{\bar{Q}} P_{\bar{Q}}^{i} c_{\bar{Q}}$, or $\left.x_{\bar{Q}}=c_{\bar{Q}}\right\}$. Then,

$$
\begin{aligned}
& \sum_{x \in \bar{B}} \varphi_{x}\left(P^{i}, P^{-i}\right)=\sum_{x_{\bar{Q}} P_{\bar{Q}}^{i} c_{\bar{Q}}} \sum_{x_{M-\bar{Q}} \in A_{M-\bar{Q}}} \varphi_{\left(x_{\bar{Q}}, x_{M-\bar{Q}}\right)}\left(P^{i}, P^{-i}\right)+\sum_{x_{M-\bar{Q}} \in A_{M-\bar{Q}}} \varphi_{\left(c_{\bar{Q}}, x_{M-\bar{Q}}\right)}\left(P^{i}, P^{-i}\right) \\
& =\sum_{x_{\bar{Q}} P_{\bar{Q}}^{i} c_{\bar{Q}}} \varphi_{\bar{Q}, x_{\bar{Q}}}\left(P^{i}, P^{-i}\right)+\varphi_{\bar{Q}, c_{\bar{Q}}}\left(P^{i}, P^{-i}\right) \\
& <\sum_{x_{\bar{Q}} P_{\bar{Q}}^{i} c_{\bar{Q}}} \varphi_{\bar{Q}, x_{\bar{Q}}}\left(\bar{P}^{i}, P^{-i}\right)+\varphi_{\bar{Q}, c_{\bar{Q}}}\left(\bar{P}^{i}, P^{-i}\right) \\
& =\sum_{x \in \bar{B}} \varphi_{x}\left(\bar{P}^{i}, P^{-i}\right)
\end{aligned}
$$

Therefore, voter $i$ manipulates at $\left(P^{i}, P^{-i}\right)$ via $\bar{P}^{i}$.

Case 2: $\varphi_{\bar{Q}, c_{\bar{Q}}}(P)>\varphi_{\bar{Q}, c_{\bar{Q}}}\left(\bar{P}^{i}, P^{-i}\right)$.

Let $\bar{w}_{\bar{Q}}$ denote $\bar{P}_{M-\bar{Q}}^{i}-$ minimal alternative. We claim that $c_{\bar{Q}}$ is the $\bar{P}_{\bar{Q}}^{i}-$ maximal alternative such that $\varphi_{\bar{Q}, c_{\bar{Q}}}\left(\bar{P}^{i}, P^{-i}\right) \neq \varphi_{\bar{Q}, c_{\bar{Q}}}\left(P^{i}, P^{-i}\right)$.

Suppose not. Then there exists $d_{\bar{Q}} \in A_{\bar{Q}}$ such that $d_{\bar{Q}} \bar{P}_{\bar{Q}}^{i} c_{\bar{Q}}$ and $\varphi_{\bar{Q}, d_{\bar{Q}}}\left(\bar{P}^{i}, P^{-i}\right) \neq$ $\varphi_{\bar{Q}, d_{\bar{Q}}}\left(P^{i}, P^{-i}\right)$. Since $P_{\bar{Q}}^{i}=\bar{P}_{\bar{Q}}^{i}$, we have that $d_{\bar{Q}} P_{\bar{Q}}^{i} c_{\bar{Q}}$ and $\varphi_{\bar{Q}, d_{\bar{Q}}}\left(\bar{P}^{i}, P^{-i}\right) \neq \varphi_{\bar{Q}, d_{\bar{Q}}}\left(P^{i}, P^{-i}\right)$, which is a contradiction to the definition of $c_{\bar{Q}}$. Therefore, for all $x_{\bar{Q}} \in A_{\bar{Q}}$ with $x_{\bar{Q}} \bar{P}_{\bar{Q}}^{i} c_{\bar{Q}}$, we have $\varphi_{\bar{Q}, x_{\bar{Q}}}\left(\bar{P}^{i}, P^{-i}\right)=\varphi_{\bar{Q}, x_{\bar{Q}}}\left(P^{i}, P^{-i}\right)$.

Now, consider $\left(c_{\bar{Q}}, \bar{w}_{M-\bar{Q}}\right)$ and $\hat{B}=B\left(\left(c_{\bar{Q}}, \bar{w}_{M-\bar{Q}}\right), \bar{P}^{i}\right)$. Since all components in $\bar{Q}$ lexicographically dominate all components in $M-\bar{Q}$, we know $\hat{B}=\left\{x \in A\right.$ : either $x_{\bar{Q}} \bar{P}_{\bar{Q}}^{i} c_{\bar{Q}}$, or $x_{\bar{Q}}=$ $\left.c_{\bar{Q}}\right\}$. Then,

$$
\begin{aligned}
\sum_{x \in \hat{B}} \varphi_{x}\left(\bar{P}^{i}, P^{-i}\right) & =\sum_{x_{\bar{Q}} \bar{P}_{\bar{Q}}^{i} c_{\bar{Q}}} \sum_{x_{M-\bar{Q}} \in A_{M-\bar{Q}}} \varphi_{\left(x_{\bar{Q}}, x_{M-\bar{Q}}\right)}\left(\bar{P}^{i}, P^{-i}\right)+\sum_{x_{M-\bar{Q}} \in A_{M-\bar{Q}}} \varphi_{\left(c_{\bar{Q}}, x_{M-\bar{Q}}\right)}\left(\bar{P}^{i}, P^{-i}\right) \\
& =\sum_{x_{\bar{Q}} \bar{P}_{\bar{Q}}^{i} c_{\bar{Q}}} \varphi_{\bar{Q}, x_{\bar{Q}}}\left(\bar{P}^{i}, P^{-i}\right)+\varphi_{\bar{Q}, c_{\bar{Q}}}\left(\bar{P}^{i}, P^{-i}\right) \\
& <\sum_{x_{\bar{Q}} \bar{P}_{\bar{Q}}^{i} c_{\bar{Q}}} \varphi_{\bar{Q}, x_{\bar{Q}}}\left(P^{i}, P^{-i}\right)+\varphi_{\bar{Q}, c_{\bar{Q}}}\left(P^{i}, P^{-i}\right) \\
& =\sum_{x \in \hat{B}} \varphi_{x}\left(P^{i}, P^{-i}\right)
\end{aligned}
$$


Hence, voter $i$ manipulates at $\left(\bar{P}^{i}, P^{-i}\right)$ via $P^{i}$. Therefore $\varphi_{\bar{Q}}\left(P^{i}, P^{-i}\right)=\varphi_{\bar{Q}}\left(\bar{P}^{i}, P^{-i}\right)$.

We say that orderings $P^{i}, \bar{P}^{i} \in\left[\mathbb{D}^{L}\right]$ are marginally equivalent if $P_{j}^{i}=\bar{P}_{j}^{i}$ for all $j \in M$. Similarly, we will say that profiles $P, \bar{P} \in\left[\mathbb{D}^{L}\right]^{N}$ are marginally equivalent if $P^{i}$ and $\bar{P}^{i}$ are marginally equivalent for all $i \in I$.

Lemma 3 Let $P \in\left[\mathbb{D}^{L}\right]^{N}, i \in I$ and $\bar{P}^{i} \in \mathbb{D}^{L}$ be such that (i) $P^{i}$ and $\bar{P}^{i}$ are marginally equivalent and (ii) if $P^{i}$ and $\bar{P}^{i}$ are lexicographic with respect to the orderings $\succ$ and $\succ$ over $M$ respectively, then $\succ$ and $\succ$ agree over all components except $j$ and $k$ where $j$ and $k$ are contiguous in $\succ$. If $\varphi(P) \neq \varphi\left(\bar{P}^{i}, P^{-i}\right)$ then $\varphi_{j}(P) \neq \varphi_{j}\left(\bar{P}^{i}, P^{-i}\right)$ and $\varphi_{k}(P) \neq \varphi_{k}\left(\bar{P}^{i}, P^{-i}\right)$.

Proof: Suppose the Lemma is false. In view of Lemma 2, we can assume that $j$ and $k$ are the lexicographic best and second best components respectively in $P^{i}$ and the lexicographic second and best components in $\bar{P}^{i}$ respectively. We have therefore assumed that $\varphi\left(P^{i}, P^{-i}\right) \neq$ $\varphi\left(\bar{P}^{i}, P^{-i}\right)$ but $\varphi_{l}\left(P^{i}, P^{-i}\right)=\varphi_{l}\left(\bar{P}^{i}, P^{-i}\right)$ for all components $l$. Let $a$ be the highest ranked alternative in $P^{i}$ such that $\varphi_{a}\left(P^{i}, P^{-i}\right) \neq \varphi_{a}\left(\bar{P}^{i}, P^{-i}\right)$. Since $\varphi$ is strategy-proof, it must be the case that $\varphi_{a}\left(P^{i}, P^{-i}\right)>\varphi_{a}\left(\bar{P}^{i}, P^{-i}\right)$. Let $Y=\left\{x \in A: x_{k} \bar{P}_{k}^{i} a_{k}\right\}=\left\{x \in A: x_{k} P_{k}^{i} a_{k}\right\}$ (since $\bar{P}_{k}^{i}=P_{k}^{i}$ ). Let $Z=\left\{x \in A: x_{k}=a_{k}\right.$ and $\left.x \bar{P}_{k}^{i} a\right\}$. Note that $Z=\left\{x \in A: x_{k}=\right.$ $a_{k}$ and $\left.x P^{i} a\right\}$ since $P^{i}$ and $\bar{P}^{i}$ are marginally equivalent orderings and the lexicographic ordering of components in $M \backslash k$ in the two orderings is also the same. Note the following

(i) $B\left(a, \bar{P}^{i}\right)=Y \cup Z \cup\{a\}$

(ii) $\varphi_{z}\left(\bar{P}^{i}, P^{-i}\right)=\varphi_{z}\left(P^{i}, P^{-i}\right)$ for all $z \in Z$ since $z \in Z$ implies that $z P^{i} a$ and $a$ is the highest-ranked alternative $P^{i}$ such that $\varphi_{a}\left(P^{i}, P^{-i}\right) \neq \varphi_{a}\left(\bar{P}^{i}, P^{-i}\right)$ and

(iii) $\sum_{\left\{x_{k}: x_{k} \bar{P}_{k}^{i} a_{k}\right\}} \varphi_{k, x_{k}}\left(\bar{P}^{i}, P^{-i}\right)=\sum_{\left\{x_{k}: x_{k} \bar{P}_{k}^{i} a_{k}\right\}} \varphi_{k, x_{k}}\left(P^{i}, P^{-i}\right)$ by virtue of our assumption that the $\varphi$ yields the same marginal probability distribution all over components.

Hence,

$$
\begin{aligned}
\sum_{x \in B\left(a, \bar{P}^{i}\right)} \varphi_{x}\left(P^{i}, P^{-i}\right) & =\sum_{x \in Y} \varphi_{x}\left(P^{i}, P^{-i}\right)+\sum_{x \in Z} \varphi_{x}\left(P^{i}, P^{-i}\right)+\varphi_{a}\left(P^{i}, P^{-i}\right) \\
& =\sum_{\left\{x_{k}: x_{k} P_{k}^{i} a_{k}\right\}} \varphi_{k, x_{k}}\left(P^{i}, P^{-i}\right)+\sum_{x \in Z} \varphi_{x}\left(P^{i}, P^{-i}\right)+\varphi_{a}\left(P^{i}, P^{-i}\right) \\
& >\sum_{\left\{x_{k}: x_{k} \bar{P}_{k}^{i} a_{k}\right\}} \varphi_{k, x_{k}}\left(\bar{P}^{i}, P^{-i}\right)+\sum_{x \in Z} \varphi_{x}\left(\bar{P}^{i}, P^{-i}\right)+\varphi_{a}\left(\bar{P}^{i}, P^{-i}\right) \\
& =\sum_{x \in Y} \varphi_{k, x_{k}}\left(\bar{P}^{i}, P^{-i}\right)+\sum_{x \in Z} \varphi_{x}\left(\bar{P}^{i}, P^{-i}\right)+\varphi_{a}\left(\bar{P}^{i}, P^{-i}\right) \\
& =\sum_{x \in B\left(a, \bar{P}^{i}\right)} \varphi_{x}\left(\bar{P}^{i}, P^{-i}\right)
\end{aligned}
$$


Consequently voter $i$ manipulates at $\left(\bar{P}^{i}, P^{-i}\right)$ via $P^{i}$ contradicting the strategy-proofness of $\varphi$.

STEP 1: The goal of this Step is to show the following. Pick an arbitrary non-empty subset $Q \subset M$. Then there exists a unanimous, strategy-proof $\operatorname{RSCF} \varphi^{Q}:\left[\mathbb{D}_{Q}^{L}\right]^{N} \rightarrow \mathcal{L}\left(A_{Q}\right)$ such that for all profiles $P \in\left[\mathbb{D}^{L}\right]^{N}$ satisfying $\tau\left(P_{M-Q}^{i}, A_{M-Q}\right)=a_{M-Q}$ for all $i \in I$, we have

1. $\left[\varphi_{x}(P)>0\right] \Rightarrow\left[x_{M-Q}=a_{M-Q}\right]$ and

2. $\varphi_{\left(x_{Q}, a_{M-Q}\right)}(P)=\varphi_{x_{Q}}^{Q}\left(P_{Q}\right)$ for all $x_{Q} \in A_{Q}$.

Thus, there exists a strategy-proof $\operatorname{RSCF} \varphi^{Q}$ defined for every non-empty set of components $Q$ with the property that whenever all voters are unanimous with respect to say $a_{M-Q} \in A_{M-Q}$, then $\varphi$ (i) puts strictly positive probability only on those alternatives whose $M-Q$ are given by $a_{M-Q}$ and (ii) the probability of an alternative $\left(a_{Q}, a_{M-Q}\right)$ in the profile $P$ is the probability given to $a_{Q}$ in the $\operatorname{RSCF} \varphi^{Q}$ in the component $Q$ induced profile $P_{Q}$. Moreover $\varphi^{Q}$ satisfies unanimity.

The arguments involved in Step 1 are generalizations of counterparts in LeBreton and Sen (1999).

The first lemma asserts that $\varphi$ satisfies a conditional unanimity property.

Lemma 4 Let $Q \subset M, P \in\left[\mathbb{D}^{L}\right]^{N}$ and $a \in A$ be such that $\tau\left(P_{M-Q}^{i}, A_{M-Q}\right)=a_{M-Q}$ for all $i \in I$. Then $\left[\varphi_{b}(P)>0\right] \Rightarrow\left[b_{M-Q}=a_{M-Q}\right]$.

Proof: Suppose that the Lemma is false. Assume that $\tau\left(P_{M-Q}^{i}, A_{M-Q}\right)=a_{M-Q}$ for all $i \in I$ but $\varphi_{b}(P)>0$ where $b_{M-Q} \neq a_{M-Q}$. For all $i \in I$, let $\bar{P}^{i} \in \mathbb{D}^{L}$ be such that (i) $\bar{P}_{k}^{i}=P_{k}^{i}$ for all $k \in M-Q$ (ii) $\tau\left(\bar{P}_{Q}^{i}, A_{Q}\right)=b_{Q}$ and (iii) all components in $Q$ lexicographically dominate all components in $M-Q$.

Pick an arbitrary voter $i$ and suppose $\varphi_{x}\left(\bar{P}^{i}, P^{-i}\right)=0$ whenever $x_{Q}=b_{Q}$. For any $k \in M-Q$, let $d_{k} \in A_{k}$ be the worst ranked element in $A_{k}$ according to $\bar{P}_{k}^{i}$ (and $P_{k}^{i}$ ). Let $\bar{B}=B\left(\left(b_{Q}, d_{M-Q}\right), \bar{P}^{i}\right)$. Since components in $Q$ lexicographically dominate those in $M-Q$ in $\bar{P}^{i}$, it follows that $c \in \bar{B} \Rightarrow\left[c_{Q}=b_{Q}\right]$. Therefore

$$
\sum_{x \in \bar{B}} \varphi_{x}\left(P^{i}, P^{-i}\right) \geq \varphi_{b}(P)>0=\sum_{x \in \bar{B}} \varphi_{x}\left(\bar{P}^{i}, P^{-i}\right) .
$$

Hence $i$ manipulates $\varphi$ at $\left(\bar{P}^{i}, P_{-i}\right)$ via $P^{i}$. Therefore, $\varphi_{\left(b_{Q}, c_{M-Q}\right)}\left(\bar{P}^{i}, P^{-i}\right)>0$ for some $c_{M-Q} \in A_{M-Q}$.

Now suppose $\varphi_{\left(b_{Q}, a_{M-Q}\right)}\left(\bar{P}^{i}, P^{-i}\right)=1$. Let $\hat{B}=B\left(\left(b_{Q}, a_{M-Q}\right), P^{i}\right)$. Note that $\left(b_{Q}, a_{M-Q}\right) P^{i} b$ since $a_{M-Q}=\tau\left(P^{i}, A_{M-Q}\right)$. Since

$$
\sum_{x \in \hat{B}} \varphi_{x}\left(P^{i}, P^{-i}\right)<\sum_{x \in \hat{B}} \varphi_{x}\left(\bar{P}^{i}, P^{-i}\right)=1
$$


voter $i$ will manipulate at $P$ via $\bar{P}^{i}$. Therefore $\varphi_{\left(b_{Q}, a_{M-Q}\right)}\left(\bar{P}^{i}, P^{-i}\right)<1$.

We can conclude from the arguments in the two previous paragraphs that there exists $c_{M-Q} \in A_{M-Q} \backslash\left\{a_{M-Q}\right\}$ such that $\varphi_{\left(b_{Q}, c_{M-Q}\right)}\left(\bar{P}^{i}, P^{-i}\right)>0$. Now pick a voter $i^{\prime} \neq i$ and replace $P^{i^{\prime}}$ in the profile $\left(\bar{P}^{i}, P^{-i}\right)$ by $\bar{P}^{i^{\prime}}$. Replicating the arguments above, we can conclude that there exists $d_{M-Q} \in A_{M-Q} \backslash\left\{a_{M-Q}\right\}$ such that $\varphi_{\left(b_{Q}, d_{M-Q}\right)}\left(\bar{P}^{i}, \bar{P}^{i^{\prime}}, \bar{P}^{-i, i^{\prime}}\right)>0$. Proceeding in this manner, it follows that $\varphi_{\left(b_{Q}, x_{M-Q}\right)}(\bar{P})>0$ where $x_{M-Q} \in A_{M-Q} \backslash\left\{a_{M-Q}\right\}$. But all voters have $\left(b_{Q}, a_{M-Q}\right)$ as their first-ranked alternative in the profile $\bar{P}$. Hence $\varphi$ violates unanimity completing the proof of the Lemma.

For every $Q \subset M$ and $a \in A$, let $\left[\mathbb{D}^{L}(a, Q)\right]^{N} \subset\left[\mathbb{D}^{L}\right]^{N}$ be the set of lexicographic profiles $P$ with the property that $\tau\left(P_{M-Q}^{i}, A_{M-Q}\right)=a_{M-Q}$ for all $i \in I$.

Lemma 5 Let $Q \subset M$ and $a \in A$. Let $\hat{P}, \bar{P} \in\left[\mathbb{D}^{L}(a, Q)\right]^{N}$ be such that $\hat{P}_{Q}=\bar{P}_{Q}$. Then $\varphi(\hat{P})=\varphi(\bar{P})$.

Proof: It follows from Lemma 4 that $\left[\varphi_{b}(\hat{P})>0\right] \Rightarrow\left[b_{M-Q}=a_{M-Q}\right]$ and $\left[\varphi_{b}(\bar{P})>0\right] \Rightarrow$ $\left[b_{M-Q}=a_{M-Q}\right]$. We first claim that $\varphi\left(\bar{P}^{i}, \hat{P}^{-i}\right)=\varphi(\hat{P})$ for an arbitrary voter $i$. Suppose this is false. Let $c_{Q}$ be the best-alternative in $A_{Q}$ according to $\hat{P}_{Q}^{i}=\bar{P}_{Q}^{i}$ such that

$$
\varphi_{\left(c_{Q}, a_{M-Q}\right)}\left(\bar{P}^{i}, \hat{P}^{-i}\right) \neq \varphi_{\left(c_{Q}, a_{M-Q}\right)}\left(\bar{P}^{i}, \hat{P}^{-i}\right) .
$$

Such an alternative $c_{Q}$ must exist. If

$$
\varphi_{\left(c_{Q}, a_{M-Q}\right)}\left(\bar{P}^{i}, \hat{P}^{-i}\right)>\varphi_{\left(c_{Q}, a_{M-Q}\right)}(\hat{P})
$$

then

$$
\sum_{x \in B\left(\left(c_{Q}, a_{M-Q}\right), \hat{P}^{i}\right)} \varphi_{x}\left(\bar{P}^{i}, \hat{P}^{-i}\right)>\sum_{x \in B\left(\left(c_{Q}, a_{M-Q}\right), \hat{P}^{i}\right)} \varphi_{x}(\hat{P})
$$

contradicting the strategy-proofness of $\varphi$. If

$$
\varphi_{\left(c_{Q}, a_{M-Q}\right)}\left(\bar{P}^{i}, \hat{P}^{-i}\right)<\varphi_{\left(c_{Q}, a_{M-Q}\right)}(\hat{P})
$$

then

$$
\sum_{x \in B\left(\left(b_{Q}, a_{M-Q}\right), \bar{P}^{i}\right)} \varphi_{x}(\hat{P})>\sum_{x \in B\left(\left(c_{Q}, a_{M-Q}\right), \bar{P}^{i}\right)} \varphi_{x}\left(\bar{P}^{i}, \hat{P}^{-i}\right)
$$

again contradicting the strategy-proofness of $\varphi$. Therefore $\varphi\left(\bar{P}^{i}, \hat{P}^{-i}\right)=\varphi(\hat{P})$. Progressively switching preferences of voters from $\hat{P}^{i}$ to $\bar{P}^{i}$ and repeatedly applying these arguments above yields $\varphi(\hat{P})=\varphi(\bar{P})$ as required.

Let $a \in A$ and $Q \subset M$. We define the function $\varphi^{a, Q}:\left[\mathbb{D}_{Q}^{L}\right]^{N} \rightarrow \mathcal{L}\left(A_{Q}\right)$ as follows: for all $P \in\left[\mathbb{D}^{L}(a, Q)\right]^{N}$ and $x_{Q} \in A_{Q}, \varphi_{x_{Q}}^{a, Q}\left(P_{Q}\right)=\varphi_{\left(x_{Q}, a_{M-Q}\right)}(P)$, where $P_{Q}$ is the induced 
profile of preferences of $P$. Thus we obtain $\varphi^{a, Q}$ by considering a profile $P \in\left[\mathbb{D}^{L}(a, Q)\right]^{N}$ and equating the probability that $\varphi^{a, Q}\left(P_{Q}\right)$ gives to every $b_{Q} \in A_{Q}$ with $\varphi_{\left(b_{j}, a_{M-Q}\right)}(P)$. A critical observation is that Lemma 5 implies that $\varphi^{a, Q}$ is well-defined. The next Lemma demonstrates that it is strategy-proof.

LEMma $6 \varphi^{a, Q}$ is strategy-proof and satisfies unanimity.

Proof: Suppose $\varphi^{a, Q}$ is not strategy-proof. Then there must exist $i \in I, P_{Q} \in\left[\mathbb{D}^{L}(a, Q)\right]^{N}$, $\bar{P}_{Q}^{i} \in\left[\mathbb{D}^{L}(a, Q)\right]$ and $b_{Q} \in A_{Q}$ such that

$$
\sum_{x_{Q} \in B\left(b_{Q}, P_{Q}^{i}\right)} \varphi_{x_{Q}}^{a, Q}\left(\bar{P}_{Q}^{i}, P_{Q}^{-i}\right)>\sum_{x_{Q} \in B\left(b_{Q}, P_{Q}^{i}\right)} \varphi_{x_{Q}}^{a, Q}\left(P_{Q}\right) .
$$

Let $\hat{P} \in\left[\mathbb{D}^{L}\right]^{N}$ be a profile and $\tilde{P}^{i} \in \mathbb{D}^{L}$ be an ordering such that (i) $\hat{P}_{Q}=P_{Q}$ (ii) $\tau\left(\hat{P}_{M-Q}^{t}, A_{M-Q}\right)=a_{M-Q}$ for all voters $t \in I$ (iii) all components in $Q$ are lexicographically dominated by those in $M-Q$ in $\hat{P}^{t}$ for all $t \in I$ (iv) $\tilde{P}_{Q}^{i}=\bar{P}_{Q}^{i}$ (v) $\tau\left(\tilde{P}_{M-Q}^{i}, A_{M-Q}\right)=a_{M-Q}$ and (vi) all components in $Q$ are lexicographically dominated by those in $M-Q$ in $\tilde{P}^{i}$.

By construction, the profiles $\hat{P},\left(\tilde{P}^{i}, \hat{P}^{-i}\right) \in\left[\mathbb{D}^{S}(a, Q)\right]^{N}$. Hence Lemma 4 implies that $\left[\varphi_{b}(\hat{P})>0\right] \Rightarrow\left[b_{M-Q}=a_{M-Q}\right]$ and $\left[\varphi_{b}\left(\tilde{P}^{i}, \hat{P}^{-i}\right)>0\right] \Rightarrow\left[b_{M-Q}=a_{M-Q}\right]$. Since components in $Q$ are lexicographically dominated by those in $M-Q$ in both $\hat{P}^{i}$ and $\tilde{P}^{i}$ and $\tau\left(\hat{P}_{M-Q}^{i}, A_{M-Q}\right)=a_{M-Q}$ we must have $B\left(\left(b_{Q}, a_{M-Q}\right), \hat{P}^{i}\right)=\left\{\left(x_{Q}, a_{M-Q}\right): x_{Q} \in B\left(b_{Q}, \hat{P}_{Q}^{i}\right)\right\}$.

Consequently

$$
\begin{aligned}
\sum_{x \in B\left(\left(b_{Q}, a_{M-Q}\right), \hat{P}^{i}\right)} \varphi_{x}\left(\tilde{P}^{i}, \hat{P}^{-i}\right) & =\sum_{x_{Q} \in B\left(b_{Q}, \hat{P}_{Q}^{i}\right)} \varphi_{x_{Q}}^{a, Q}\left(\tilde{P}_{Q}^{i}, \hat{P}_{Q}^{-i}\right) \\
& =\sum_{x_{Q} \in B\left(b_{Q}, P_{Q}^{i}\right)} \varphi_{x_{Q}}^{a, Q}\left(\tilde{P}_{Q}^{i}, \hat{P}_{Q}^{-i}\right) \\
& >\sum_{x_{Q} \in B\left(b_{Q}, P_{Q}^{i}\right)} \varphi_{x_{Q}}^{a, Q}\left(P_{Q}\right) \\
& =\sum_{x \in B\left(\left(b_{Q}, a_{M-Q}\right), \hat{P}^{i}\right)} \varphi_{x}(\hat{P}) .
\end{aligned}
$$

contradicting the strategy-proofness of $\varphi$. Therefore $\varphi^{a, Q}$ is strategy-proof.

Now let $P$ be a profile such that $P_{Q} \in\left[\mathbb{D}^{L}(a, Q)\right]^{N}$ be a profile such that all voters are unanimous with respect to components in $Q$, i.e. suppose $\tau\left(P_{Q}^{i}, A_{Q}\right)=b_{Q}$ for some $b_{Q} \in A_{Q}$. Clearly $\tau\left(P^{i}, A\right)=\left(b_{Q}, a_{M-Q}\right)$. Since $\varphi$ satisfies unanimity, $\varphi(P)=\left(b_{Q}, a_{M-Q}\right)$ which implies that $\varphi^{a, Q}\left(P_{Q}\right)=b_{Q}$. Therefore $\varphi^{a, Q}$ satisfies unanimity.

Lemma $7 \varphi^{a, Q}$ does not depend on a i.e. $\varphi^{a, Q}=\varphi^{b, Q}$ for all $b \in A$. 
Proof: Given any $a, b \in A, Q \subset M$ and $P_{Q} \in\left[\mathbb{D}_{Q}^{L}\right]^{N}$, consider following two profiles $\bar{P} \in\left[\mathbb{D}^{L}(a, Q)\right]^{N}$ and $\hat{P} \in\left[\mathbb{D}^{L}(b, Q)\right]^{N}$ such that (i) $\bar{P}_{Q}=\hat{P}_{Q}=P_{Q}$, (ii) $Q$ lexicographically dominates $M-Q$ in $\bar{P}^{i}$ for all $i \in I$ and (iii) $Q$ lexicographically dominates $M-Q$ in $\hat{P}^{i}$ for all $i \in I$. Then, by Lemma 4 , we have that for all $x_{Q} \in A_{Q}$,

$$
\begin{aligned}
& \varphi_{Q, x_{Q}}\left(\bar{P}^{i}, \bar{P}^{-i}\right) \equiv \sum_{x_{M-Q} \in A_{M-Q}} \varphi_{\left(x_{Q}, x_{M-Q}\right)}\left(\bar{P}^{i}, \bar{P}^{-i}\right)=\varphi_{\left(x_{Q}, a_{M-Q}\right)}\left(\bar{P}^{i}, \bar{P}^{-i}\right) \\
& \varphi_{Q, x_{Q}}\left(\hat{P}^{i}, \hat{P}^{-i}\right) \equiv \sum_{x_{M-Q} \in A_{M-Q}} \varphi_{\left(x_{Q}, x_{M-Q}\right)}\left(\hat{P}^{i}, \hat{P}^{-i}\right)=\varphi_{\left(x_{Q}, b_{M-Q}\right)}\left(\hat{P}^{i}, \hat{P}^{-i}\right)
\end{aligned}
$$

Since $\bar{P} \in\left[\mathbb{D}^{L}(a, Q)\right]^{N}$ and $\hat{P} \in\left[\mathbb{D}^{L}(b, Q)\right]^{N}$ we know that $\varphi_{x_{Q}}^{a, Q}\left(P_{Q}\right)=\varphi_{\left(x_{Q}, a_{M-Q}\right)}\left(\bar{P}^{i}, \bar{P}^{-i}\right)$ and $\varphi_{x_{Q}}^{b, Q}\left(P_{Q}\right)=\varphi_{\left(x_{Q}, b_{M-Q}\right)}\left(\hat{P}^{i}, \hat{P}^{-i}\right)$ for all $x_{Q} \in A_{Q}$. Hence, $\varphi_{x_{Q}}^{a, Q}\left(P_{Q}\right)=\varphi_{Q, x_{Q}}\left(\bar{P}^{i}, \bar{P}^{-i}\right)$ and $\varphi_{x_{Q}}^{b, Q}\left(P_{Q}\right)=\varphi_{Q, x_{Q}}\left(\hat{P}^{i}, \hat{P}^{-i}\right)$ for all $x_{Q} \in A_{Q}$.

Since $\bar{P}_{Q}^{i}=\hat{P}_{Q}^{i}$, and $Q$ lexicographically dominates $M-Q$ in both $\bar{P}_{Q}^{i}$ and $\hat{P}_{Q}^{i}$, we can apply Lemma 2 to obtain the following conclusion: $\varphi_{Q}\left(\bar{P}^{i}, \bar{P}^{-i}\right)=\varphi_{Q}\left(\hat{P}^{i}, \bar{P}^{-i}\right)$. Applying the same argument to all other voters, we have that $\varphi_{Q}\left(\bar{P}^{i}, \bar{P}^{-i}\right)=\varphi_{Q}\left(\hat{P}^{i}, \hat{P}^{-i}\right)$. Consequently, $\varphi_{x_{Q}}^{a, Q}\left(P_{Q}\right)=\varphi_{x_{Q}}^{b, Q}\left(P_{Q}\right)$ for all $x_{Q} \in A_{Q}$.

This concludes Step 1.

Step 2: The goal of this step is to show the following: Let $I=\{1,2\}$ and let $\varphi$ : $\left[\mathbb{D}^{L}\right]^{2} \rightarrow \mathcal{L}(A)$ be a strategy-proof RSCF satisfying unanimity. Then $\varphi$ is a generalized random dictatorship. Throughout Step 2, we assume that $\varphi$ is a two-voter RSCF defined on the domain of lexicographic preferences that is strategy-proof and satisfies unanimity.

For any $P \in\left[\mathbb{D}^{L}\right]^{2}$, the Top Product Set at $P$ or $T P S(P)$ is defined as follows:

$$
T P S(P) \equiv\left[\left\{\tau\left(P_{1}^{1}, A_{1}\right)\right\} \cup\left\{\tau\left(P_{1}^{2}, A_{1}\right)\right\}\right] \times \ldots \times\left[\left\{\tau\left(P_{m}^{1}, A_{m}\right)\right\} \cup\left\{\tau\left(P_{m}^{2}, A_{m}\right)\right\}\right] .
$$

We say that $\varphi$ satisfies the TPS Property if

$$
\sum_{a \in T P S(P)} \varphi_{a}(P)=1 \quad \text { for all } P \in\left[\mathbb{D}^{L}\right]^{2} .
$$

Lemma 8 y satisfies the TPS Property.

Proof: Suppose the Lemma is false. Then there exists $P \in\left[\mathbb{D}^{L}\right]^{2}, a, b, c \in A$ and $j \in M$ such that $\varphi_{c}(P)>0$ and $\tau\left(P^{1}, A\right)=a, \tau\left(P^{2}, A\right)=b$ and $c_{j} \notin\left\{a_{j}, b_{j}\right\}$. We consider two mutually exhaustive cases.

Case 1: Component $j$ is the lexicographically worst component in $P^{1}$ and $P^{2}$. 
Claim 1: If $\varphi_{j, x_{j}}(P)>0$ where $x_{j} \notin\left\{a_{j}, b_{j}\right\}$, then $a_{j} P_{j}^{1} x_{j} P_{j}^{1} b_{j}$ and $b_{j} P_{j}^{2} x_{j} P_{j}^{2} a_{j}$.

Suppose that the Claim is false. Assume without loss of generality that $b_{j} P_{j}^{1} x_{j}$ and $\varphi_{j, x_{j}}(P)>0$. Consider $\bar{P}^{1} \in \mathbb{D}^{L}$ such that (i) $\bar{P}_{M-j}^{1}=P_{M-j}^{1}$ (ii) $\tau\left(\bar{P}_{j}^{1}, A_{j}\right)=b_{j}$ and (iii) component $j$ is lexicographically worst in $\bar{P}^{1}$. By Lemmas 2 and 4 , we have that $\sum_{z_{j} \in A_{j}} \varphi_{\left(y_{M-j}, z_{j}\right)}\left(P^{1}, P^{2}\right)=\sum_{z_{j} \in A_{j}} \varphi_{\left(y_{M-j}, z_{j}\right)}\left(\bar{P}^{1}, P^{2}\right)=\varphi_{\left(y_{M-j}, b_{j}\right)}\left(\bar{P}^{1}, P^{2}\right)$.

Since $\varphi_{\left(y_{M-j}, x_{j}\right)}\left(P^{1}, P^{2}\right)>0$, it must be the case that

$$
\begin{aligned}
\sum_{z_{j} P_{j}^{1} x_{j}} \varphi_{\left(y_{M-j}, z_{j}\right)}\left(P^{1}, P^{2}\right) & <\sum_{z_{j} \in A_{j}} \varphi_{\left(y_{M-j}, z_{j}\right)}\left(P^{1}, P^{2}\right) \\
& =\varphi_{\left(y_{M-j}, b_{j}\right)}\left(\bar{P}^{1}, P^{2}\right) .
\end{aligned}
$$

Since $b_{j} P_{j}^{1} x_{j}$, Lemma 4 implies that $\sum_{z_{j} P_{j}^{1} x_{j}} \varphi_{\left(y_{M-j}, z_{j}\right)}\left(\bar{P}^{1}, P^{2}\right)=\varphi_{\left(y_{M-j}, b_{j}\right)}\left(\bar{P}^{1}, P^{2}\right)$. Therefore, $\sum_{z_{j} P_{j}^{1} x_{j}} \varphi_{\left(y_{M-j}, z_{j}\right)}\left(P^{1}, P^{2}\right)<\sum_{z_{j} P_{j}^{1} x_{j}} \varphi_{\left(y_{M-j}, z_{j}\right)}\left(\bar{P}^{1}, P^{2}\right)$.

By virtue of the fact that component $j$ is lexicographically worst in $P^{1}$, it follows that $\bar{B}=B\left(\left(y_{M-j}, x_{j}\right), P^{1}\right) \backslash\left\{\left(y_{M-j}, x_{j}\right)\right\}=\left\{z \in A: z_{M-j} P_{M-j}^{1} y_{M-j}\right.$ or $z_{M-j}=y_{M-j}$ and $\left.z_{j} P_{j}^{1} x_{j}\right\}$. An application of Lemma 2 yields

$$
\begin{aligned}
\sum_{z \in \bar{B}} \varphi_{z}\left(P^{1}, P^{2}\right) & =\sum_{z_{M-j} P_{M-j}^{1} y_{M-j}} \sum_{z_{j} \in A_{j}} \varphi_{\left(z_{M-j}, z_{j}\right)}\left(P^{1}, P^{2}\right)+\sum_{z_{j} P_{j}^{1} x_{j}} \varphi_{\left(y_{M-j}, z_{j}\right)}\left(P^{1}, P^{2}\right) \\
& =\sum_{z_{M-j} P_{M-j}^{1} y_{M-j}} \sum_{z_{j} \in A_{j}} \varphi_{\left(z_{M-j}, z_{j}\right)}\left(\bar{P}^{1}, P^{2}\right)+\sum_{z_{j} P_{j}^{1} x_{j}} \varphi_{\left(y_{M-j}, z_{j}\right)}\left(P^{1}, P^{2}\right) \\
& <\sum_{z_{M-j} P_{M-j}^{1} y_{M-j}} \sum_{z_{j} \in A_{j}} \varphi_{\left(z_{M-j}, z_{j}\right)}\left(\bar{P}^{1}, P^{2}\right)+\sum_{z_{j} P_{j}^{1} x_{j}} \varphi_{\left(y_{M-j}, z_{j}\right)}\left(\bar{P}^{1}, P^{2}\right) \\
& =\sum_{z \in \bar{B}} \varphi_{z}\left(\bar{P}^{1}, P^{2}\right)
\end{aligned}
$$

Therefore, voter 1 will manipulate at $\left(P^{1}, P^{2}\right)$ via $\bar{P}^{1}$. This proves Claim 1.

In view of Claim 1 , we can assume that $a_{j} P_{j}^{1} c_{j} P_{j}^{1} b_{j}$ and $b_{j} P_{j}^{2} c_{j} P_{j}^{2} a_{j}$. Let $\bar{P}^{1} \in \mathbb{D}^{L}$ be such that (i) $\bar{P}_{M-j}^{1}=P_{M-j}^{1}$ (ii) $\tau\left(\bar{P}_{j}^{1}, A_{j}\right)=a_{j}$ and $b_{j}$ is ranked second in $A_{j}$ according to $\bar{P}_{j}^{1}$ and (iii) $j$ is lexicographically worst in $\bar{P}^{i}$. We claim that $\varphi_{j, a_{j}}(P)=\varphi_{j, a_{j}}\left(\bar{P}^{1}, P^{2}\right)$. If $\varphi_{j, a_{j}}(P)<\varphi_{j, a_{j}}\left(\bar{P}^{1}, P^{2}\right)$, then we can construct an argument analogous to the one above to show that 1 manipulates at $P$ via $\bar{P}^{1}$. If the reverse is true, 1 manipulates at $\left(\bar{P}^{1}, P^{2}\right)$ via $P^{1}$. It follows from our earlier arguments that $\varphi_{j, z_{j}}\left(\bar{P}^{1}, P^{2}\right)=0$ for all $z_{j} \neq a_{j}, b_{j}$. Since $\varphi_{j, c_{j}}(P)>0$ and $\varphi_{j, a_{j}}(P)=\varphi_{j, a_{j}}\left(\bar{P}^{1}, P^{2}\right)$, we must have $\varphi_{j, b_{j}}(P)<\varphi_{j, b_{j}}\left(\bar{P}^{1}, P^{2}\right)$.

Now construct $\bar{P}^{2} \in \mathbb{D}^{L}$ be such that (i) $\bar{P}_{M-j}^{2}=P_{M-j}^{2}$ (ii) $\tau\left(\bar{P}_{j}^{2}, A_{j}\right)=b_{j}$ and $a_{j}$ is ranked second in $A_{j}$ according to $\bar{P}_{j}^{2}$ and (iii) component $j$ is lexicographically worst in $\bar{P}^{2}$. From our earlier arguments $\varphi_{j, z_{j}}(\bar{P})=0$ for all $z_{j} \neq a_{j}, b_{j}$ and $\varphi_{j, b_{j}}(\bar{P})=\varphi_{j, b_{j}}\left(\bar{P}^{1}, P^{2}\right)$. Therefore $\varphi_{j, a_{j}}(\bar{P})=\varphi_{j, a_{j}}\left(\bar{P}^{1}, P^{2}\right)$. Hence $\varphi_{j, b_{j}}(\bar{P})>\varphi_{j, b_{j}}(P)$. 
Now consider $\varphi_{j}\left(P^{1}, \bar{P}^{2}\right)$. Using the same arguments as before, we can deduce that $\varphi_{j, b_{j}}(P)=\varphi_{j, b_{j}}\left(P^{1}, \bar{P}^{2}\right), \varphi_{j, a_{j}}(P)<\varphi_{j, a_{j}}\left(P^{1}, \bar{P}^{2}\right)$ and $\varphi_{j, z_{j}}\left(P^{1}, \bar{P}^{2}\right)=0$ for all $z_{j} \neq a_{j}, b_{j}$. Furthermore $\varphi_{j, b_{j}}(\bar{P})=\varphi_{j, b_{j}}\left(P^{1}, \bar{P}^{2}\right), \varphi_{j, a_{j}}(\bar{P})=\varphi_{j, a_{j}}\left(P^{1}, \bar{P}^{2}\right)$ and $\varphi_{j, z_{j}}(\bar{P})=0$ for all $z_{j} \neq$ $a_{j}, b_{j}$. Hence $\varphi_{j, b_{j}}(\bar{P})=\varphi_{j, b_{j}}(P)$ contradicting our earlier conclusion that $\varphi_{j, b_{j}}(\bar{P})>\varphi_{j, b_{j}}(P)$. This completes Case 1.

Case 2: Case 1 does not hold. Assume without loss of generality that $j$ is not the lexicographically worst component in $P^{1}$. Let $S$ and $T$ denote the set of components lexicographically worse than $j$ and lexicographically better than $j$ respectively. Let $\bar{P}^{1} \in \mathbb{D}^{L}$ such that (i) $\bar{P}_{T \cup\{j\}}^{1}=P_{T \cup\{j\}}^{1}$, (ii) the set of components lexicographically better than $j$ in $\bar{P}^{1}$ is $T$ and (iii) $\tau\left(\bar{P}_{S}^{1}, A_{S}\right)=b_{s}$.

Suppose $\varphi_{j}\left(\bar{P}^{1}, P^{2}\right) \neq \varphi_{j}\left(\hat{P}^{1}, P^{2}\right)$. Since $j \in T \cup\{j\}$, it must be true that $\varphi_{T \cup\{j\}}\left(\bar{P}^{1}, P^{2}\right) \neq \varphi_{T \cup\{j\}}\left(\hat{P}^{1}, P^{2}\right)$. Let $\left(x_{T}, x_{j}\right)$ be $\bar{P}_{T \cup\{j\}}^{1}$-maximal such that $\varphi_{T \cup\{j\},\left(x_{T}, x_{j}\right)}\left(\bar{P}^{1}, P^{2}\right) \neq \varphi_{T \cup\{j\},\left(x_{T}, x_{j}\right)}\left(\hat{P}^{1}, P^{2}\right)$. Since $\tau\left(\bar{P}_{S}^{1}, A_{S}\right)=\tau\left(\hat{P}_{S}^{1}, A_{S}\right)=\tau\left(P_{S}^{2}, A_{S}\right)=$ $b_{S}$, we can apply Lemma 4 , we know that $\varphi_{T \cup\{j\},\left(x_{T}, x_{j}\right)}\left(\bar{P}^{1}, P^{2}\right)=\varphi_{\left(x_{T}, x_{j}, b_{S}\right)}\left(\bar{P}^{1}, P^{2}\right)$ and $\varphi_{T \cup\{j\},\left(x_{T}, x_{j}\right)}\left(\hat{P}^{1}, P^{2}\right)=\varphi_{\left(x_{T}, x_{j}, b_{S}\right)}\left(\hat{P}^{1}, P^{2}\right)$. Hence, $\varphi_{\left(x_{T}, x_{j}, b_{S}\right)}\left(\bar{P}^{1}, P^{2}\right) \neq \varphi_{\left(x_{T}, x_{j}, b_{S}\right)}\left(\hat{P}^{1}, P^{2}\right)$.

Assume first that $\varphi_{\left(x_{T}, x_{j}, b_{S}\right)}\left(\bar{P}^{1}, P^{2}\right)<\varphi_{\left(x_{T}, x_{j}, b_{S}\right)}\left(\hat{P}^{1}, P^{2}\right)$. Since in $\bar{P}^{1}, T$ lexicographically dominates $j$ and $j$ lexicographically dominates $S$, we know that $\bar{B}=B\left(\left(x_{T}, x_{j}, b_{S}\right), \bar{P}^{1}\right)=$ $\left\{z \in A:\left(z_{T}, z_{j}\right) \bar{P}_{T \cup\{j\}}^{1}\left(x_{T}, x_{j}\right)\right\} \cup\left\{\left(x_{T}, x_{j}, b_{S}\right)\right\}$. Then,

$$
\begin{aligned}
\sum_{z \in \bar{B}} \varphi_{z}\left(\bar{P}^{1}, P^{2}\right) & =\sum_{\left(z_{T}, z_{j}\right) \bar{P}_{T \cup\{j\}}^{1}\left(x_{T}, x_{j}\right)} \sum_{z_{S} \in A_{S}} \varphi_{\left(z_{T}, z_{j}, z_{S}\right)}\left(\bar{P}^{1}, P^{2}\right)+\varphi_{\left(x_{T}, x_{j}, b_{S}\right)}\left(\bar{P}^{1}, P^{2}\right) \\
& <\sum_{\left(z_{T}, z_{j}\right) \bar{P}_{T \cup\{j\}}^{1}\left(x_{T}, x_{j}\right)} \sum_{z_{S} \in A_{S}} \varphi_{\left(z_{T}, z_{j}, z_{S}\right)}\left(\hat{P}^{1}, P^{2}\right)+\varphi_{\left(x_{T}, x_{j}, b_{S}\right)}\left(\hat{P}^{1}, P^{2}\right) \\
& =\sum_{z \in \bar{B}} \varphi_{z}\left(\hat{P}^{1}, P^{2}\right)
\end{aligned}
$$

Therefore, voter 1 will manipulate at $\left(\bar{P}^{1}, P^{2}\right)$ via $\hat{P}^{1}$.

Next, assume that $\varphi_{\left(x_{T}, x_{j}, b_{S}\right)}\left(\bar{P}^{1}, P^{2}\right)>\varphi_{\left(x_{T}, x_{j}, b_{S}\right)}\left(\hat{P}^{1}, P^{2}\right)$. Since $T$ lexicographically dominates $S$ and $S$ lexicographically dominates $j$ in $\hat{P}^{1}$, we know that

$\hat{B}=B\left(\left(x_{T}, x_{j}, b_{S}\right), \hat{P}^{1}\right)=\left\{z \in A: z_{T} \hat{P}_{T}^{1} x_{T}\right.$, or $z_{T}=x_{T}, z_{S}=b_{S}$ and $\left.z_{j} \hat{P}_{j}^{1} x_{j}\right\} \cup$ $\left\{\left(x_{T}, x_{j}, b_{S}\right)\right\}$.

Since $\hat{P}^{1}$ and $\bar{P}^{1}$ agree on the lexicographic order over $T, T$ lexicographically dominates $M-T$ in both $\hat{P}^{1}$ and $\bar{P}^{1}$ and $\hat{P}_{k}^{1}=\bar{P}_{k}^{1}$ for all $k \in M$, we can conclude that $\hat{P}_{T}^{1}=\bar{P}_{T}^{1}$. Furthermore, since $T$ lexicographically dominates $M-T$ in both $\hat{P}^{1}$ and $\bar{P}^{1}$, Lemma 2 implies $\varphi_{T}\left(\hat{P}^{1}, P^{2}\right)=\varphi_{T}\left(\bar{P}^{1}, P^{2}\right)$ and hence $\sum_{z_{T} \hat{P}_{T}^{1} x_{T}} \varphi_{T, z_{T}}\left(\hat{P}^{1}, P^{2}\right)=\sum_{z_{T} \hat{P}_{T}^{1} x_{T}} \varphi_{T, z_{T}}\left(\bar{P}^{1}, P^{2}\right)$. Also, for any $z_{j} \in A_{j}$ with $z_{j} \hat{P}_{j}^{1} x_{j}$, we know that $z_{j} \bar{P}_{j}^{1} x_{j}$ since $\bar{P}_{j}=\hat{P}_{j}^{1}$. Hence $\left(x_{T}, z_{j}\right) \bar{P}_{T \cup\{j\}}^{1}\left(x_{T}, x_{j}\right)$. Therefore, according to the definition of $\left(x_{T}, x_{j}\right)$ in $\bar{P}^{1}$, we have $\varphi_{T \cup\{j\},\left(x_{T}, z_{j}\right)}\left(\bar{P}^{1}, P^{2}\right)=$ 
$\varphi_{T \cup\{j\},\left(x_{T}, z_{j}\right)}\left(\hat{P}^{1}, P^{2}\right)$ for all $z_{j} \hat{P}_{j}^{1} x_{j}$. Furthermore, since $\tau\left(\bar{P}_{S}^{1}, A_{S}\right)=\tau\left(\hat{P}_{S}^{1}, A_{S}\right)=\tau\left(P_{S}^{2}, A_{S}\right)=$ $b_{S}$, Lemma 4 implies that $\varphi_{\left(x_{T}, z_{j}, b_{S}\right)}\left(\bar{P}^{1}, P^{2}\right)=\varphi_{T \cup\{j\},\left(x_{T}, z_{j}\right)}\left(\bar{P}^{1}, P^{2}\right)=\varphi_{T \cup\{j\},\left(x_{T}, z_{j}\right)}\left(\hat{P}^{1}, P^{2}\right)=$ $\varphi_{\left(x_{T}, z_{j}, b_{S}\right)}\left(\hat{P}^{1}, P^{2}\right)$ for all $z_{j} \hat{P}_{j}^{1} x_{j}$.

Hence

$$
\begin{aligned}
& \sum_{z \in \hat{B}} \varphi_{z}\left(\hat{P}^{1}, P^{2}\right) \\
= & \sum_{z_{T} \hat{P}_{T}^{1} x_{T}} \sum_{z_{M-T} \in A_{M-T}} \varphi_{\left(z_{T}, z_{M-T}\right)}\left(\hat{P}^{1}, P^{2}\right)+\sum_{z_{j} \hat{P}_{j}^{1} x_{j}} \varphi_{\left(x_{T}, z_{j}, b_{S}\right)}\left(\hat{P}^{1}, P^{2}\right)+\varphi_{\left(x_{T}, x_{j}, b_{S}\right)}\left(\hat{P}^{1}, P^{2}\right) \\
< & \sum_{z_{T} \hat{P}_{T}^{1} x_{T}} \sum_{z_{M-T} \in A_{M-T}} \varphi_{\left(z_{T}, z_{M-T}\right)}\left(\bar{P}^{1}, P^{2}\right)+\sum_{z_{j} \hat{P}_{j}^{1} x_{j}} \varphi_{\left(x_{T}, z_{j}, b_{S}\right)}\left(\bar{P}^{1}, P^{2}\right)+\varphi_{\left(x_{T}, x_{j}, b_{S}\right)}\left(\bar{P}^{1}, P^{2}\right) \\
= & \sum_{z \in \hat{B}} \varphi_{z}\left(\bar{P}^{1}, P^{2}\right) .
\end{aligned}
$$

Therefore, voter 1 will manipulate at $\left(\hat{P}^{1}, P^{2}\right)$ via $\bar{P}^{1}$. In conclusion, $\varphi_{j}\left(\bar{P}^{1}, P^{2}\right)=$ $\varphi_{j}\left(\hat{P}^{1}, P^{2}\right)$ which establishes the claim.

Similarly we can find $\hat{P}^{2} \in \mathbb{D}^{L}$ where $j$ is lexicographically worst and $\varphi_{j}(\hat{P})=\varphi_{j}(P)$. Therefore $\varphi_{j, c_{j}}(\hat{P})>0$. Note that $\tau\left(\hat{P}_{j}^{i}, A_{j}\right) \neq c_{j}$ for $i=1,2$. Hence we are in the situation described in Case 1 and we can use the same arguments to show that $\varphi_{j, c_{j}}(\hat{P})>0$ is not possible.

According to our next lemma, the outcome of a strategy-proof RSCF in the two-voter case is identical across marginally equivalent profiles.

Lemma 9 Let $P, \bar{P} \in\left[\mathbb{D}^{L}\right]^{2}$ be marginally equivalent profiles. Then $\varphi(P)=\varphi(\bar{P})$.

Proof: Let $P, \bar{P} \in\left[\mathbb{D}^{S}\right]^{2}$ be marginally equivalent profiles. Suppose $\tau\left(P^{1}\right)=a$ and $\tau\left(P^{2}\right)=$ $b$. Since $P$ and $\bar{P}$ are marginally equivalent it follows that $\tau\left(\bar{P}^{1}\right)=a$ and $\tau\left(\bar{P}^{2}\right)=b$. Moreover $a_{j} P_{j}^{1} b_{j}, a_{j} \bar{P}_{j}^{1} b_{j}$ and $b_{j} P_{j}^{2} a_{j}, b_{j} \bar{P}_{j}^{2} a_{j}$ for all $j \in M$ whenever $a_{j}$ and $b_{j}$ are distinct. According to Lemma 8, the support of the lotteries $\varphi(P)$ and $\varphi(\bar{P})$ are the same and equal to the set $\left\{a_{1}, b_{1}\right\} \times \ldots \times\left\{a_{m}, b_{m}\right\}$. We will show that these lotteries are in fact, equal to each other. We prove this by induction on the number of components.

The result follows from the Gibbard random dictatorship result in the case where $m=1$. Assume now that the following is true.

Induction Hypothesis (IH): Suppose there are $t \geq 2$ components. Let $P, \bar{P} \in\left[\mathbb{D}^{L}\right]^{2}$ be marginally equivalent profiles. Then $\varphi(P)=\varphi(\bar{P})$ for all unanimous and strategy-proof $\operatorname{RSCFs} \varphi\left[\mathbb{D}^{L}\right]^{2} \rightarrow \mathcal{L}(A)$.

We will show that Lemma 9 holds in the case of $t+1$ components. We will prove this in two steps. 
Claim 2: Suppose that there are $t+1$ components. Let $P, \bar{P} \in\left[\mathbb{D}^{L}\right]^{2}$ be two profiles such that there exists a component assumed without loss of generality to be component $t+1$ and

1. $\tau\left(P_{t+1}^{1}\right)=\tau\left(P_{t+1}^{2}\right)=x_{t+1}$ and $\tau\left(\bar{P}_{t+1}^{1}\right)=\tau\left(\bar{P}_{t+1}^{2}\right)=y_{t+1}$

2. $P_{k}^{i}=\bar{P}_{k}^{i}$ for $i=1,2$ and all components $k=1, \ldots t$.

Then $\varphi_{\left(a, x_{t+1}\right)}(P)=\varphi_{\left(a, y_{t+1}\right)}(\bar{P})$ for all $t$-component alternatives $a$.

Let $P_{-(t+1)}$ and $\bar{P}_{-(t+1)}$ denote the profiles of preferences induced over all components other than $t+1$ by the profiles $P$ and $\bar{P}$ respectively. Observe that $P_{-(t+1)}$ and $\bar{P}_{-(t+1)}$ are marginally equivalent over all components other than $t+1$ by 2 above. Applying Lemma 7, we know that there exists a $t$ component strategy-proof RSCF $\varphi^{\prime}$ such that

(i) $\left[\varphi_{\left(a, a_{t+1}\right)}(P)>0\right] \Rightarrow\left[a_{t+1}=x_{t+1}\right]$

(ii) $\left[\varphi_{\left(a, a_{t+1}\right)}(\bar{P})>0\right] \Rightarrow\left[a_{t+1}=y_{t+1}\right]$

(iii) $\varphi_{\left(a, x_{t+1}\right)}(P)=\varphi_{a}^{\prime}\left(P_{-(t+1)}\right)$

(iv) $\varphi_{\left(a, y_{t+1}\right)}(\bar{P})=\varphi_{a}^{\prime}\left(\bar{P}_{-(t+1)}\right)$

Note that $I H$ implies that $\varphi_{a}^{\prime}\left(P_{-(t+1)}\right)=\varphi_{a}^{\prime}\left(\bar{P}_{-(t+1)}\right)$. Therefore $\varphi_{\left(a, x_{t+1}\right)}(P)=\varphi_{\left(a, y_{t+1}\right)}(\bar{P})$. This completes Claim 2.

We now complete the proof of the induction step. In view of Claim 2 the only case that needs to be considered is the one where $\tau\left(P^{1}\right)=a$ and $\tau\left(P^{2}\right)=b$ and $a_{j} \neq b_{j}$ for all $j=1, \ldots t+1$. Suppose that $\varphi\left(P^{1}, P^{2}\right) \neq \varphi\left(\bar{P}^{1}, P^{2}\right)$. (Recall that $P^{1}$ and $\bar{P}^{1}$ are marginally equivalent.) There must exist $x, y \in T P S(P)=T P S(\bar{P})$ such that $x P^{1} y$ and $y \bar{P}^{1} x$. We claim that there must exist at least two components say $j$ and $k$ such that $x_{j} \neq y_{j}$ and $x_{k} \neq y_{k}$. Of course, at least one such component is required; otherwise $x=y$. Suppose there exists exactly one such component, say $j$. Then separability of preference orderings would imply that the marginal preferences over component $j$ have switched between $P^{1}$ and $\bar{P}^{1}$ contradicting our hypothesis that $P^{1}$ and $\bar{P}^{1}$ are marginally equivalent.

From Lemma 3 we know that there exist $j, k \in M, a_{j} \in A_{j}$ and $a_{k} \in A_{k}$ such that $\varphi_{j, a_{j}}\left(\bar{P}^{1}, P^{2}\right)>\varphi_{j, a_{j}}(P)$ and $\varphi_{k, a_{k}}\left(\bar{P}^{1}, P^{2}\right)<\varphi_{k, a_{k}}(P) .{ }^{4}$

Consider the second ranked alternative $x$ in $P^{2}$. There must exist a unique component say $l$ such that $x_{l} \neq b_{l}$ and $x_{j}=b_{j}$ for all $j \neq l$. In fact, we can assume w.l.o.g that $x_{l}=a_{l}$. We consider two cases.

Case 1: $j \neq l$. Let $\tilde{P}^{1}$ be an ordering such that (i) component $j$ is lexicographically best and (ii) $\tau\left(\tilde{P}^{1}, A\right)=a$. If $\varphi_{j, a_{j}}\left(\tilde{P}^{1}, P^{2}\right)<\varphi_{j, a_{j}}\left(\bar{P}^{1}, P^{2}\right)$, then voter 1 will manipulate at

\footnotetext{
${ }^{4}$ The proof of this claim requires consideration of several cases. These are routine and we omit the details.
} 
$\left(\tilde{P}^{1}, P^{2}\right)$ via $\bar{P}^{1}$. Therefore $\varphi_{j, a_{j}}\left(\tilde{P}^{1}, P^{2}\right) \geq \varphi_{j, a_{j}}\left(\bar{P}^{1}, P^{2}\right)$. Let $\hat{P}^{1}$ be an ordering where (i) $j$ is the lexicographic best component (ii) $\tau\left(\hat{P}_{l}^{1}, A_{l}\right)=b_{l}$ and (iii) $\hat{P}_{M-l}^{1}=P_{M-l}^{1}$. By strategyproofness, $\varphi_{j, a_{j}}\left(\hat{P}^{1}, P_{2}\right)=\varphi_{j, a_{j}}\left(\tilde{P}^{1}, P_{2}\right)$. Hence $\varphi_{j, a_{j}}\left(\hat{P}^{1}, P_{2}\right)>\varphi_{j, a_{j}}(P)$. Observe that at the profile $\left(\hat{P}^{1}, P^{2}\right)$, both voters have a common maximal alternative $b_{l}$ for component $l$.

Let $\tilde{P}^{2}$ be a lexicographic ordering where $j$ and $l$ are the best and the worst components respectively and $\tau\left(\tilde{P}^{2}, A\right)=b$. Using the same argument as before, $\varphi_{j, b_{j}}\left(P^{1}, \tilde{P}^{2}\right) \geq \varphi_{j, b_{j}}(P)$ i.e. by Lemma $4, \varphi_{j, a_{j}}\left(P^{1}, \tilde{P}^{2}\right) \leq \varphi_{j, a_{j}}(P)$. Let $\hat{P}^{2}$ be a lexicographic ordering such that (i) components $j$ and $l$ are the best and worst respectively (ii) $\tau\left(\hat{P}_{l}^{2}, A_{l}\right)=a_{l}$ and (iii) $\hat{P}_{M-l}^{2}=$ $P_{M-l}^{2}$. As before, $\varphi_{j, b_{j}}\left(P^{1}, \tilde{P}^{2}\right)=\varphi_{j, b_{j}}\left(P^{1}, \hat{P}^{2}\right)$. Then Lemma 8 implies $\varphi_{j, a_{j}}\left(P^{1}, \tilde{P}^{2}\right)=$ $\varphi_{j, a_{j}}\left(P^{1}, \hat{P}^{2}\right)$ so that $\varphi_{j, a_{j}}\left(P^{1}, \hat{P}^{2}\right) \leq \varphi_{j, a_{j}}(P)$.

Observe that at the profile $\left(P^{1}, \hat{P}^{2}\right)$, both voters have a common maximal alternative $a_{l}$ for component $l$. By Lemma 4 and Claim 2 above (in the proof of this Lemma), we must have $\varphi_{j, a_{j}}\left(\hat{P}^{1}, P^{2}\right)=\varphi_{j, a_{j}}\left(P^{1}, \hat{P}^{2}\right)$. However, we have shown that $\varphi_{j, a_{j}}\left(\hat{P}^{1}, P_{2}\right)>\varphi_{j, a_{j}}(P) \geq$ $\varphi_{a_{j}}\left(P^{1}, \hat{P}^{2}\right)$. We have a contradiction.

Case 2: $j=l$. Let $\tilde{P}^{2}$ be a lexicographic ordering where component $k$ is lexicographically best. Using a similar argument as before we have $\varphi_{k, a_{k}}\left(\bar{P}^{1}, \tilde{P}^{2}\right) \leq \varphi_{k, a_{k}}\left(\bar{P}^{1}, P^{2}\right)$. Let $\hat{P}^{2}$ be a lexicographic ordering where $k$ is the best and $\tau\left(\hat{P}_{l}^{2}, A_{l}\right)=a_{l}$. By strategy-proofness, $\varphi_{k, a_{k}}\left(P^{1}, \hat{P}^{2}\right)=\varphi_{k, a_{k}}\left(P^{1}, \tilde{P}^{2}\right)$. Hence $\varphi_{k, a_{k}}\left(P^{1}, \hat{P}^{2}\right)<\varphi_{k, a_{k}}(P)$. Observe that at the profile $\left(P^{1}, \hat{P}^{2}\right)$, both voters have a common maximal alternative $a_{l}$ for component $l$.

Let $\tilde{P}^{1}$ be a lexicographic ordering where $k$ and $l$ are the best and the worst components respectively. Using the same argument as before, $\varphi_{k, a_{k}}\left(\tilde{P}^{1}, P^{2}\right) \geq \varphi_{k, a_{k}}(P)$. Let $\hat{P}^{1}$ be a lexicographic ordering such that component $k$ and $l$ are the best and worst respectively and $\tau\left(\hat{P}_{l}^{1}, A_{l}\right)=b_{l}$. As before, $\varphi_{k, a_{k}}\left(\tilde{P}^{1}, P^{2}\right)=\varphi_{k, a_{k}}\left(\hat{P}^{1}, P^{2}\right)$ so that $\varphi_{k, a_{k}}\left(\hat{P}^{1}, P^{2}\right) \geq \varphi_{k, a_{k}}(P)$.

Observe that at the profile $\left(\hat{P}^{1}, P^{2}\right)$, both voters have a common maximal alternative $b_{l}$ for component $l$. By the same argument as at the end of Case 1, it follows that $\varphi_{k, a_{k}}\left(\hat{P}^{1}, P^{2}\right)=$ $\varphi_{k, a_{k}}\left(P^{1}, \hat{P}^{2}\right)$. However, we have shown that $\varphi_{k, a_{k}}\left(\hat{P}^{1}, P^{2}\right)>\varphi_{k, a_{k}}(P) \geq \varphi_{k, a_{k}}\left(P^{1}, \hat{P}^{2}\right)$. We have a contradiction.

The next Lemma establishes the following: the probability distribution on the top product set of the two voters depends on the maximal alternatives of the voters.

Lemma 10 For all $P, \bar{P} \in\left[\mathbb{D}^{L}\right]^{2}$ such that $\tau\left(P_{k}^{i}, A_{k}\right)=\tau\left(\bar{P}_{k}^{i}, A_{k}\right)$ for all $k \in M$ and $i \in I$, we have $\varphi(P)=\varphi(\bar{P})$.

Proof: Let $Q \subseteq M$ be such that for all $j \in Q, \tau\left(P_{j}^{1}, A_{j}\right) \neq \tau\left(P_{j}^{2}, A_{j}\right)$ and for all $k \in$ $M-Q, \tau\left(P_{k}^{1}, A_{k}\right)=\tau\left(P_{k}^{2}, A_{k}\right)$. (It is possible that $M-Q=\emptyset$.) Assume w.l.o.g that (i) $\tau\left(P_{j}^{1}, A_{j}\right)=\tau\left(\bar{P}_{j}^{1}, A_{j}\right)=x_{j}$ and $\tau\left(P_{j}^{2}, A_{j}\right)=y_{j}$ for all $j \in Q$ and (ii) $\tau\left(P_{k}^{1}, A_{k}\right)=$ $\tau\left(\bar{P}_{k}^{1}, A_{k}\right)=\tau\left(P_{k}^{2}, A_{k}\right)=z_{k}$ for all $k \in M-Q$. Therefore, $\operatorname{TPS}\left(P^{1}, P^{2}\right)=\operatorname{TP} S\left(\bar{P}^{1}, P^{2}\right)=$ $\prod_{j \in Q}\left\{x_{j}, y_{j}\right\} \times \prod_{k \in M-Q}\left\{z_{k}\right\}$. 
We claim that $P^{1}$ and $\bar{P}^{2}$ agree on $T P S\left(P^{1}, P^{2}\right)$. By Lemma 9 , we can assume that $P^{1}$ and $\bar{P}^{1}$ have a common lexicographic ordering over components which has the property that $Q$ lexicographically dominates $M-Q$. Given $a, b \in T P S\left(P^{1}, P^{2}\right)$, let $k$ be lexicographically maximal component such that $a_{k} \neq b_{k}$. Then, it is evident that $k \in Q$. Since $a_{k}, b_{k} \in$ $\left\{x_{k}, y_{k}\right\}, x_{k} P_{k}^{1} y_{k}$ and $x_{k} \bar{P}_{k}^{1} y_{k}$, if $a_{k} P_{k}^{1} b_{k}$, it must be the case that $a P^{1} b, a_{k}=x_{k}$ and $b_{k}=y_{k}$. Hence, $a_{k} \bar{P}_{k}^{1} b_{k}$. Furthermore, since $P^{1}$ and $\bar{P}^{1}$ agree on the lexicographic order, it is true that $a \bar{P}^{1} b$. Symmetrically, we also show that $\left[a \bar{P}^{1} b\right] \Rightarrow\left[a P^{1} b\right]$ for all $a, b \in T P S\left(P^{1}, P^{2}\right)$.

Next, to simplify the notation, we assume that $\operatorname{TP} S\left(P^{1}, P^{2}\right)=\left\{a^{k}\right\}_{k=1}^{T}$ for some integer $T>0$ such that $a^{k} P^{1} a^{k+1}, k=1, \ldots, T-1$. Hence, $a^{k} \bar{P}^{1} a^{k+1}, k=1, \ldots, T-1$. By Lemma 8, we know that for all $x \in A-T P S\left(P^{1}, P^{2}\right), \varphi_{x}\left(P^{1}, P^{2}\right)=\varphi_{x}\left(\bar{P}^{1}, P^{2}\right)=0$. Therefore, for all $k=2, \ldots, T, \sum_{x \in B\left(a^{k}, P^{1}\right)} \varphi_{x}\left(P^{1}, P^{2}\right)=\sum_{s=1}^{k} \varphi_{a^{s}}\left(P^{1}, P^{2}\right)$ and $\sum_{x \in B\left(a^{k}, \bar{P}^{1}\right)} \varphi_{x}\left(\bar{P}^{1}, P^{2}\right)=$ $\sum_{s=1}^{k} \varphi_{a^{s}}\left(\bar{P}^{1}, P^{2}\right)$. Meanwhile, since $P^{1}$ and $\bar{P}^{2}$ agree on $\operatorname{TP} S\left(P^{1}, P^{2}\right)$, we also have that for all $k=2, \ldots, T, \sum_{x \in B\left(a^{k}, \bar{P}^{1}\right)} \varphi_{x}\left(P^{1}, P^{2}\right)=\sum_{s=1}^{k} \varphi_{a^{s}}\left(P^{1}, P^{2}\right)$ and $\sum_{x \in B\left(a^{k}, P^{1}\right)} \varphi_{x}\left(\bar{P}^{1}, P^{2}\right)=$ $\sum_{s=1}^{k} \varphi_{a^{s}}\left(\bar{P}^{1}, P^{2}\right)$. Therefore, by strategy-proofness, we have that for all $k=2, \ldots, T$, $\sum_{s=1}^{k} \varphi_{a^{s}}\left(P^{1}, P^{2}\right)=\sum_{s=1}^{k} \varphi_{a^{s}}\left(\bar{P}^{1}, P^{2}\right)$. Furthermore, strategy-proofness also implies that $\varphi_{a^{1}}\left(P^{1}, P^{2}\right)=\varphi_{a^{1}}\left(\bar{P}^{1}, P^{2}\right)$. Therefore, $\varphi_{a^{k}}\left(P^{1}, P^{2}\right)=\varphi_{a^{k}}\left(\bar{P}^{1}, P^{2}\right)$ for all $k=1, \ldots, T$. Now, we conclude that for all $x \in T P S\left(P^{1}, P^{2}\right), \varphi_{x}\left(P^{1}, P^{2}\right)=\varphi_{x}\left(\bar{P}^{1}, P^{2}\right)$ and for all $x \in$ $A-T P S\left(P^{1}, P^{2}\right), \varphi_{x}\left(P^{1}, P^{2}\right)=\varphi_{x}\left(\bar{P}^{1}, P^{2}\right)=0$. Consequently, $\varphi\left(P^{1}, P^{2}\right)=\varphi\left(\bar{P}^{1}, P^{2}\right)$.

Applying the same argument to voter 2 , we can show that $\varphi\left(\bar{P}^{1}, P^{2}\right)=\varphi\left(\bar{P}^{1}, \bar{P}^{2}\right)$. Therefore, $\varphi\left(P^{1}, P^{2}\right)=\varphi\left(\bar{P}^{1}, \bar{P}^{2}\right)$.

Lemma 11 Let $j \in M, P \in\left[\mathbb{D}^{L}\right]^{2}, \bar{P}^{i} \in \mathbb{D}^{L}$ and $\left(x_{j}, z_{M-j}\right),\left(y_{j}, z_{M-j}\right) \in A$ be such that (i) $\tau\left(P_{k}^{i}, A_{k}\right)=\tau\left(\bar{P}_{k}^{i}, A_{k}\right)$ for all $k \neq j$ (ii) $\tau\left(P_{j}^{i}, A_{j}\right)=x_{j}$ and $\tau\left(\bar{P}_{j}^{i}, A_{j}\right)=y_{j}$ and (iii) $\left(x_{j}, z_{M-j}\right) \in T P S(P)$. Then

$$
\varphi_{\left(x_{j}, z_{M-j}\right)}\left(\bar{P}^{i}, P^{-i}\right)+\varphi_{\left(y_{j}, z_{M-j}\right)}\left(\bar{P}^{i}, P^{-i}\right)=\varphi_{\left(x_{j}, z_{M-j}\right)}(P)+\varphi_{\left(y_{j}, z_{M-j}\right)}(P) .
$$

Moreover

$$
\varphi_{\left(d_{j}, z_{M-j}\right)}\left(\bar{P}^{i}, P^{-i}\right)=\varphi_{\left(d_{j}, z_{M-j}\right)}(P) \text { for all } d_{j} \notin\left\{x_{j}, y_{j}\right\} .
$$

Proof: In view of Lemmas 8, 9 and 10 we can assume without loss of generality that (i) $j$ is the lexicographically worst component in $P^{i}$ and $\bar{P}^{i}$ (ii) $P_{M-j}^{i}=\bar{P}_{M-j}^{i}$ for all $k \neq j$ and (iii) $\left(x_{j}, z_{M-j}\right)$ and $\left(y_{j}, z_{M-j}\right)$ are contiguous in $P^{i}$. Suppose $\left(a_{j}, z_{M-j}\right) P^{i}\left(x_{j}, z_{M-j}\right)$ where $a_{j} \neq y_{j}$. Since $j$ is the lexicographically worst component and $\left(x_{j}, z_{M-j}\right)$ and $\left(y_{j}, z_{M-j}\right)$ are contiguous it follows that $\left(a_{j}, z_{M-j}\right) \bar{P}^{i}\left(y_{j}, z_{M-j}\right)$. Similarly $\left(a_{j}, z_{M-j}\right) \bar{P}^{i}\left(y_{j}, z_{M-j}\right) \Rightarrow$ $\left(a_{j}, z_{M-j}\right) P^{i}\left(x_{j}, z_{M-j}\right)$. Now suppose $\left(a_{j}, b_{M-j}\right) P^{i}\left(x_{j}, z_{M-j}\right)$ where $b_{M-j} \neq z_{M-j}$. From our assumptions, $b_{M-j} P_{M-j}^{i} z_{M-j}$. Hence $b_{M-j} \bar{P}_{M-j}^{i} z_{M-j}$ and $\left(a_{j}, b_{M-j}\right) \bar{P}^{i}\left(y_{j}, z_{M-j}\right)$. Similarly, $\left(a_{j}, b_{M-j}\right) \bar{P}^{i}\left(y_{j}, z_{M-j}\right)$ implies $\left(a_{j}, b_{M-j}\right) P^{i}\left(x_{j}, z_{M-j}\right)$. Hence $\bar{P}^{i}$ is a feasible local switch of $\left(x_{j}, z_{M-j}\right)$ and $\left(y_{j}, z_{M-j}\right)$. The result now follows from Lemma 1. 
To show the second part of the Lemma, note that $\varphi_{\left(d_{j}, z_{M-j}\right)}\left(\bar{P}^{i}, P^{-i}\right)=\varphi_{\left(d_{j}, z_{M-j}\right)}(P)=0$ if $d_{j} \neq \tau\left(P_{j}^{-i}, A_{j}\right)$. Suppose $d_{j}=\tau\left(P_{j}^{-i}, A_{j}\right)$. Again, using Lemmas 8 and 9 , we can assume that $d_{j}$ is ranked third in both $P_{j}^{i}$ and $\bar{P}_{j}^{i}$. This implies that $B\left(\left(d_{j}, z_{M-j}\right), P^{i}\right)=$ $B\left(\left(d_{j}, z_{M-j}\right), \bar{P}^{i}\right)$. Now strategy-proofness implies that $\varphi_{\left(d_{j}, z_{M-j}\right)}\left(\bar{P}^{i}, P^{-i}\right)=\varphi_{\left(d_{j}, z_{M-j}\right)}(P)$.

We now complete the proof of Step 2. Let $P \in\left[\mathbb{D}^{L}\right]^{2}$ be such that $\tau\left(P^{1}\right)=a$ and $\tau\left(P^{2}\right)=b$ where $a_{j} \neq b_{j}$ for all $j \in M$. Pick an arbitrary $\underline{i} \in I^{m}$ and let $\gamma(\underline{i})=\varphi_{x}(P)$ where $\chi(x, P)=\underline{i}$. Since the maximal alternatives of the two voters for each component are distinct, there exists a unique $\underline{i} \in I^{m}$ for every $x \in T P S(P)$ such that $\chi(x, P)=\{\underline{i}\}$. Therefore

$$
\sum_{\underline{i} \in I^{m}} \gamma(\underline{i})=\sum_{x \in T P S(P)} \varphi_{x}(P)=1
$$

where the second equality follows from the fact that $\varphi$ satisfies the TPS property (Lemma 8).

Now consider $j \in M$ and $\bar{P}^{1} \in \mathbb{D}$ such that $\tau\left(\bar{P}_{j}^{1}, A_{j}\right)=c_{j} \neq b_{j}$ and $\tau\left(\bar{P}_{k}^{1}, A_{k}\right)=a_{k}$ for all $k \neq j$. Let $x \in \operatorname{TPS}(P)$. Observe that $\left[\underline{i} \in \chi\left(\left(a_{j}, x_{M-j}\right), P\right)\right] \Leftrightarrow\left[\underline{i} \in \chi\left(\left(c_{j}, x_{M-j}\right),\left(\bar{P}^{1}, P^{2}\right)\right]\right.$. Now applying Lemma 11 and the fact that $\left(c_{j}, x_{M-j}\right) \notin T P S(P)$ and $\left(a_{j}, x_{M-j}\right) \notin T P S\left(\bar{P}^{1}, P^{2}\right)$, we conclude that $\varphi_{\left(a_{j}, x_{M-j}\right)}(P)=\varphi_{\left(c_{j}, x_{M-j}\right)}\left(\bar{P}^{1}, P^{2}\right)$. Using this and the second part of Lemma 11, it follows that $\varphi_{x}\left(\bar{P}^{1}, P^{2}\right)=\sum_{\underline{i} \in \chi\left(x,\left(\bar{P}^{1}, P^{2}\right)\right)} \gamma(\underline{i})$.

Now consider the case where $\tau\left(\bar{P}_{j}^{1}, A_{j}\right)=b_{j}$. Let $x \in T P S(P)$. Observe that $[\underline{i} \in$ $\left.\chi\left(\left(a_{j}, x_{M-j}\right), P\right) \cup \chi\left(\left(b_{j}, x_{M-j}\right), P\right)\right] \Leftrightarrow\left[\underline{i} \in \chi\left(\left(b_{j}, x_{M-j}\right),\left(\bar{P}^{1}, P^{2}\right)\right]\right.$. Now applying Lemma 11 and noting the fact that $\left(a_{j}, x_{M-j}\right) \notin T P S\left(\bar{P}^{1}, P^{2}\right)$, we have

$$
\varphi_{\left(b_{j}, x_{M-j}\right)}\left(\bar{P}^{1}, P^{2}\right)=\varphi_{\left(a_{j}, x_{M-j}\right)}(P)+\varphi_{\left(b_{j}, x_{M-j}\right)}(P) .
$$

Once again, we have $\varphi_{x}\left(\bar{P}^{1}, P^{2}\right)=\sum_{\underline{i} \in \chi\left(x,\left(\bar{P}^{1}, P^{2}\right)\right)} \gamma(\underline{i})$ for all $x \in A$. Progressively replacing the maximal alternative of each component in voter 1 and voter 2's preferences and noting that the previous expression holds at all profiles along the sequence, we conclude that the expression holds for all profiles $P$. This establishes generalized random dictatorship for the case $N=2$ and completes Step 2.

Step 3: Let $N>2$ be an integer. We assume the following:

Induction Hypothesis $\left(I H^{*}\right)$. For all integers $K<N$, if $\varphi:\left[\mathbb{D}^{L}\right]^{K} \rightarrow \mathcal{L}(A)$ is strategy-proof and satisfies unanimity, then it is a generalized random dictatorship.

Let $\varphi:\left[\mathbb{D}^{L}\right]^{N} \rightarrow \mathcal{L}(A)$ be a strategy-proof RSCF satisfying unanimity. We will show that it is a generalized random dictatorship. 
Let $I^{\prime}=\left\{1^{\prime}, 3, \ldots, N\right\}$ be a set of $N-1$ voters where voters $3, \ldots, N$ are the same voters as in the set $I$. Voter $1^{\prime}$ is a voter obtained by "cloning" voters 1 and 2 in $I$. Define a RSCF over the voter set $I^{\prime}, g:\left[\mathbb{D}^{L}\right]^{N-1} \rightarrow \mathcal{L}(A)$ as follows:

$$
g\left(P^{1^{\prime}}, P^{3} \ldots, P^{N}\right)=\varphi\left(P^{1}, P^{1}, P^{3}, \ldots, P^{N}\right) \text { for all } P^{1}, P^{3}, \ldots, P^{N} \in \mathbb{D}^{L}
$$

It is clear that $g$ satisfies unanimity.

Claim 3: $g$ is strategy-proof.

Fix an arbitrary voter profile in $I^{\prime},\left(P^{1^{\prime}}, P^{3}, \ldots, P^{N}\right) \in\left[\mathbb{D}^{L}\right]^{N-1}$ and let $\bar{P}^{1} \in \mathbb{D}^{L}$. Let $a \in A$. Since $\varphi$ is strategy-proof

$$
\begin{aligned}
\sum_{x \in B\left(a, P^{1^{\prime}}\right)} g_{x}\left(P^{1^{\prime}}, P^{3}, \ldots, P^{N}\right) & =\sum_{x \in B\left(a, P^{1^{\prime}}\right)} \varphi_{x}\left(P^{1}, P^{1}, \ldots, P^{N}\right) \\
& \geq \sum_{x \in B\left(a, P^{1^{\prime}}\right)} \varphi_{x}\left(\bar{P}^{1}, P^{1}, \ldots, P^{N}\right) \\
& \geq \sum_{x \in B\left(a, P^{1^{\prime}}\right)} \varphi_{x}\left(\bar{P}^{1}, \bar{P}^{1}, \ldots, P^{N}\right) \\
& =\sum_{x \in B\left(a, P^{1^{\prime}}\right)} g_{x}\left(\bar{P}^{1^{\prime}}, P^{3}, \ldots, P^{N}\right) .
\end{aligned}
$$

Note that inequalities 1 and 2 follow from the strategy-proofness of $\varphi$.

Since $a$ was arbitrary, voter $1^{\prime}$ cannot manipulate in $g$. If voters $3, \ldots, N$ can manipulate $g$, they can also manipulate $\varphi$. Hence $g$ is strategy-proof establishing Claim 3.

It follows from Induction Hypothesis* that $g$ is a generalized random dictatorship. Let $\gamma^{g}$ be the function associated with $g$. We will write $\underline{i}^{g} \in I^{\prime m}$ for a voter sequence where $\underline{i}_{j}^{g} \in I^{\prime}$ for all $j \in M$. Hence

$$
g_{x}\left(P^{1^{\prime}}, P^{2}, \ldots, P^{N}\right)=\sum_{\underline{i}^{g} \in \chi\left(x,\left(P^{1^{\prime}}, P^{3} \ldots P^{N}\right)\right)} \gamma^{g}\left(\underline{i}^{g}\right)
$$

for all $x \in A$.

Let $T \subset M$. Let $\hat{i}_{-T}$ be an assignment of voters in $\{3, \ldots, n\}$ to components in $M-T$. In particular $\hat{i}_{-T}(k)$ is the voter in $\{3, \ldots, n\}$ assigned to component $k \in M-T$.

We shall let $\hat{i}(T)$ denote the voter sequence where voter $1^{\prime}$ is assigned to all components in $T$ and $\hat{i}_{-T}(k)$ is assigned to components $k \in M-T$. Thus $\hat{i}(T)$ assigns $1^{\prime}$ to components in $T$ and agrees with $\hat{i}_{-T}$ on $M-T$.

An extra piece of notation will be useful. Let $b \in A$ and $P \in\left[\mathbb{D}^{L}\right]^{N}$. Let

$$
Z(b, P, T)=\left\{\underline{i} \in \chi(b, P) \text { and } \underline{i}_{k} \notin\{1,2\} \text { for some } k \in T\right\}
$$


In other words, $Z(b, P, T)$ is the set of voter sequences in $I^{m}$ consistent with outcome $b$ and profile $P$ such that neither voter 1 nor 2 appear for some component in the set $T$.

Fix $T$ and $\hat{i}(T)$. Pick an arbitrary $x_{-T} \in A_{M-T}$. We say that $P \in\left[\mathbb{D}^{L}\right]^{N}$ is an $\left(\hat{i}(T), x_{-T}\right)$ extension of $\left(P_{T}^{1}, P_{T}^{2}\right)$ if

(i) $P$ induces $\left(P_{T}^{1}, P_{T}^{2}\right)$ for voters 1 and 2 and component set $T$.

(ii) $x_{k}=\tau\left(P_{k}^{\hat{i}(T)_{k}}\right) \neq \tau\left(P_{k}^{r}\right)$ for all $r \neq \hat{i}(T)_{k}$ and $k \in M-T$.

(iii) $\tau\left(P_{k}^{1}\right)=\tau\left(P_{k}^{2}\right)$ for all $k \in M-T$.

We proceed in two steps.

Step $\alpha$ : For all $\hat{i}(T)$ such that $\gamma^{g}(\hat{i}(T))=0$, we let $\gamma\left(\underline{i}(T), \hat{i}(T)_{-T}\right)=0$ for all $\underline{i}(T)$ such that $\underline{i}(T)_{k} \in\{1,2\}$ for all $k \in T$.

Step $\beta$ : We consider $\hat{i}(T)$ such that $\gamma^{g}(\hat{i}(T)) \neq 0$, i.e. the $\hat{i}(T)$ 's remaining after Step $\alpha$ has been completed. Our strategy here is to define a two-voter RSCF $h$ for voters 1 and 2 over component set $T$ depending on $\hat{i}(T)$. We do so inductively on the size of $T$.

Consider $T$ such that $|T|=1$ Fix $x_{-T} \in A_{M-T}$. Define a two-voter, one-component probabilistic rule $h^{T}$ as follows: for all profiles $\left(P_{T}^{1}, P_{T}^{2}\right)$,

$$
h_{a_{T}}^{\hat{i}(T)}\left(P_{T}^{1}, P_{T}^{2}\right)=\frac{1}{\gamma^{g}(\hat{i}(T))}\left[\varphi_{\left(a_{T}, x_{-T}\right)}(P)-\sum_{\underline{i} \in Z\left(\left(a_{T}, x_{-T}\right), P, T\right)} \gamma^{g}(\underline{i})\right]
$$

where $P$ is an $\left(\hat{i}(T), x_{-T}\right)$ extension of $\left(P_{T}^{1}, P_{T}^{2}\right)$.

We will show that $h^{\hat{i}(T)}$ is a RSCF, i.e. all the relevant probabilities are non-negative and add up to one. Moreover, it is strategy-proof and satisfies unanimity. Using Step 2 for two-person, one-component RSCFs, we will deduce that $h^{\hat{i}(T)}$ is a random dictatorship with

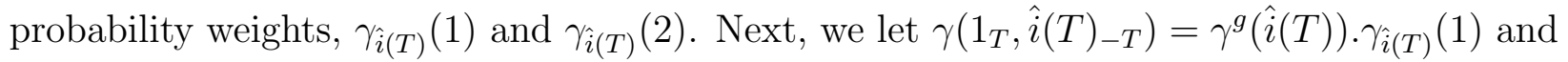
$\gamma\left(2_{T}, \hat{i}(T)_{-T}\right)=\gamma^{g}(\hat{i}(T)) \cdot \gamma_{\hat{i}(T)}(2)$. Here $\left(1_{T}, \hat{i}(T)_{-T}\right)$ refers to a voter sequence where voter 1 appears for component $T$ while the rest of the components agree with $\hat{i}(T)_{-T}$. A similar interpretation holds for $\left(2_{T}, \hat{i}(T)_{-T}\right)$. For all $\underline{i} \in I^{m}$ such that $\underline{i}_{k} \notin\{1,2\}$ for all $k \in M$, let $\gamma(\underline{i})=\gamma^{g}(\underline{i})$. We conduct this procedure with respect to all $\hat{i}(T)$ such that $|T|=1$ and obtain the corresponding $\gamma$ 's.

In the next step, we consider $\hat{i}(T)$ such that $|T|=2$. Once again, we define a two-voter, two-component probabilistic rule $h^{T}$ as follows: for all profiles $\left(P_{T}^{1}, P_{T}^{2}\right)$,

$$
h_{a_{T}}^{\hat{i}(T)}\left(P_{T}^{1}, P_{T}^{2}\right)=\frac{1}{\gamma^{g}(\hat{i}(T))}\left[\varphi_{\left(a_{T}, x_{-T}\right)}(P)-\sum_{\underline{i} \in Z\left(\left(a_{T}, x_{-T}\right), P, T\right)} \gamma(\underline{i})\right]
$$

where $P$ is an $\left(\hat{i}(T), x_{-T}\right)$ extension of $\left(P_{T}^{1}, P_{T}^{2}\right)$. 
Note that we are only using the $\gamma$ 's that we have constructed in the earlier step. We will again show that $h^{\hat{i}(T)}$ is a RSCF, is strategy-proof and satisfies unanimity. Appealing to Step 2, we deduce that $h^{\hat{i}(T)}$ is a generalized random dictatorship with associated weights $\gamma_{\hat{i}(T)}(\underline{i}(T))$ where $\underline{i}(T)_{k} \in\{1,2\}$ for all $k \in T$. Thus, $\underline{i}(T)$ is a voter sequence for the components in set $T$ where each element of the sequence is either 1 or 2 . We let $\gamma\left(\underline{i}(T), \hat{i}(T)_{-T}\right)=\gamma^{g}(\hat{i}(T)) \cdot \gamma_{\hat{i}(T)}(\underline{i}(T))$. Once again, we conduct this procedure with respect to all $\hat{i}(T)$ such that $|T|=2$ and obtain the corresponding $\gamma$ 's.

It is clear that we can continue this process till $|T|=m$. In the general step, we consider $\hat{i}(T)$ with $|T|=k+1$ after having applied the procedure for all $\hat{i}(T)$ with $|T| \leq k$ and obtained the corresponding $\gamma^{\prime}$ s. The probabilistic rule $h^{\hat{i}(T)}$ is obtained as follows: for all profiles $\left(P_{T}^{1}, P_{T}^{2}\right)$,

$$
h_{a_{T}}^{\hat{i}(T)}\left(P_{T}^{1}, P_{T}^{2}\right)=\frac{1}{\gamma^{g}(\hat{i}(T))}\left[\varphi_{\left(a_{T}, x_{-T}\right)}(P)-\sum_{\underline{i} \in Z\left(\left(a_{T}, x_{-T}\right), P, T\right)} \gamma(\underline{i})\right]
$$

where $P$ is an $\left(\hat{i}(T), x_{-T}\right)$ extension of $\left(P_{T}^{1}, P_{T}^{2}\right)$.

Note once again that the $\gamma$ 's in the bracketed term in the RHS of the equation above only uses the $\gamma$ 's obtained in the previous steps. Eventually, we will obtain $\gamma(\underline{i})$ for all $\underline{i} \in I^{m}$.

We will proceed in two steps.

Step A. Consider $T$ such that $|T|=1$. We show that $h^{\hat{i}^{g}(T)}$ as defined in Equation 3 is an RSCF. In addition, it is strategy-proof and satisfies unanimity. By virtue of Step 2, $h^{\hat{i}^{g}(T)}$ is a random dictatorship. Finally we show that this random dictatorship is (appropriately) independent of the extension.

Step B. Suppose that for all $T$ such that $|T|<k, h^{\hat{i}(T)}$ as defined in Equation 5 is an RSCF which is strategy-proof, satisfies unanimity and (appropriately) independent of the extension. Then, we show that the same is true for $h^{\hat{i}(T)}$ for $|T|=k+1$.

Step A: Let $T=\{j\}$. Note that $h^{\hat{i}^{g}(T)}$ is a one-component rule (on component $j$ ) because all components other than $j$ are fixed by the extension procedure. The claim that $h^{\hat{i}^{g}(\{j\})}$ is a RSCF follows immediately from Lemmas 4 and 5 in Sen (2011). Moreover, it is strategyproof and satisfies unanimity. It follows from Step 2 that $h^{\hat{i}(\{j\})}$ is a $j$-component random dictatorship for players 1 and 2 . Denote these probability weights by $\gamma_{\hat{i}^{g}(\{j\})}(1)$ and $\gamma_{\hat{i}^{g}(\{j\})}(2)$. Note that these weights depend (amongst other things) on $x_{-\{M-j\}}$ chosen for the extension.

LEMMA 12 The probability weights $\gamma_{\hat{i}^{g}(\{j\})}(1)$ and $\gamma_{\hat{i}^{g}(\{j\})}(2)$ do not depend on the $x_{-\{M-j\}}$ chosen for the extension. 
Proof: Suppose that the lemma is false, i.e. suppose that there exists $x_{-\{j\}}, y_{-\{j\}} \in A_{M-\{j\}}$ with associated probability weights $\gamma(1), \gamma(2)$ and $\gamma^{\prime}(1), \gamma^{\prime}(2)$ where $\gamma(1) \neq \gamma^{\prime}(1)$. ${ }^{5}$ Assume without loss of generality that $\gamma(1)>\gamma(2)$. Assume further that $x_{M-\{j\}}$ and $y_{M-\{j\}}$ differ over a single component, say $k$. For convenience, let voter $\hat{i}^{g}(\{j\})_{k}$ be denoted as voter $s$. In other words, voter $s, s \neq 1^{\prime}$ is the voter corresponding to component $k$ in the voter sequence $\hat{i}^{g}$. Let $P \in\left[\mathbb{D}^{L}\right]^{N}$ such that

(i) $\tau\left(P_{k}^{s}, A_{k}\right)=x_{k}$.

(ii) The second-ranked alternative in the $k$-th component ranking $P_{k}^{s}$ is $y_{k}$.

(iii) $k$ is the lexicographic worst component in $P^{s}$.

(iv) $\tau\left(P_{j}^{1}, A_{j}\right)=\tau\left(P_{j}^{s}, A_{j}\right)=a_{j} \neq b_{j}=\tau\left(P_{j}^{2}, A_{j}\right)$.

(v) $P$ is an $\left(\hat{i}^{g}(\{j\}), x_{M-j}\right)$ extension of $\left(P_{j}^{1}, P_{j}^{2}\right)$.

Let $\tau\left(P^{s}, A\right)=\left(a_{j}, x_{k}, d_{M-\{j, k\}}\right)$ for some $d_{M-\{j, k\}} \in A_{M-\{j, k\}}$. From (ii) and (iii) above, it is clear that the second ranked alternative in $P^{s}$ is $\left(a_{j}, y_{k}, d_{M-\{j, k\}}\right)$. (Note that $x_{M-\{j\}}=\left(x_{k}, d_{M-\{j, k\}}\right)$ and $\left.y_{M-\{j\}}=\left(y_{k}, d_{M-\{j, k\}}\right)\right)$. Since $P$ is an $\left(\hat{i}^{g}(\{j\}), x_{M-j}\right)$ extension of $\left(P_{j}^{1}, P_{j}^{2}\right)$, it follows from straightforward algebraic manipulation and strategy-proofness of $\varphi$ (for details, see Sen (2011)) that the total probability on the first and second ranked alternatives in $P^{s}$ in the profile $P$ is

$$
\gamma(1) \cdot \gamma^{g}\left(\hat{i}^{g}\right)+\sum_{\underline{i} \in Z\left(\left(a_{j}, x_{k}, d_{M-\{j, k\}}\right), P,\{j\}\right)} \gamma^{g}(\underline{i})+\sum_{\left.\underline{i} \in Z\left(\left(a_{j}, y_{k}, d_{M-\{j, k\}}\right)\right), P,\{j\}\right)} \gamma^{g}(\underline{i}) .
$$

Now suppose $s$ changes her announcement to $\bar{P}^{s}$ where the first and second ranked alternatives are interchanged. This is feasible because $k$ is the lexicographic worst component. If $\left(\bar{P}^{s}, P^{-s}\right)$ is not an extension of $\left(P_{j}^{1}, P_{j}^{2}\right)$, there is nothing to be proved. Suppose, on the other hand that it is an extension. By virtue of earlier arguments, the total probability on the first and second ranked alternatives in $P^{s}$ in the profile $P$ is

$$
\gamma^{\prime}(1) \cdot \gamma^{g}\left(\hat{i}^{g}\right)+\sum_{\underline{i} \in Z\left(\left(a_{j}, x_{k}, d_{M-\{j, k\}}\right), P,\{j\}\right)} \gamma^{g}(\underline{i})+\sum_{\left.\underline{i} \in Z\left(\left(a_{j}, y_{k}, d_{M-\{j, k\}}\right)\right), P,\{j\}\right)} \gamma^{g}(\underline{i}) .
$$

Since $\gamma(1) \neq \gamma^{\prime}(1)$ by assumption, it follows that expression $6 \neq$ expression 7 contradicting the strategy-proofness of $\varphi$. Progressively changing the components of $x_{M-j}$ to $y_{M-j}$ and applying the argument at every stage completes the proof.

REMARK: Note that the argument in the Lemma above also works for the case where voter $s$ in the construction above, is one of the voters 1 or 2 . The only case that remains is when

\footnotetext{
${ }^{5}$ We drop the subscripts and superscripts for convenience.
} 
voter $s \neq 1,2$ and the maximal alternative in a component $k$ other than $j$ for either voter 1 or 2 coincides with the maximal element in component $k$ for $s$. We do not have to deal with this situation in Steps $A$ or $B$ - we therefore address it in the final step of the proof.

Step B: Fix $T \subset M$ and $\hat{i}(T)$ such that $|T|=k+1$. Following the procedure outlined previously, we have obtained probability weights $\gamma\left(\underline{i}(T), \hat{i}(T)_{-T}\right)$, for all $T$ such that $|T| \leq k$. As described earlier, $\underline{i}(T)$ is a voter sequence over components in $T$ where element of the sequence is either 1 or 2 . Thus, $\left(\underline{i}(T), \hat{i}(T)_{-T}\right)$ is a voter sequence that is either 1 or 2 for all components in $T$ and agrees with $\hat{i}(T)$ in $M-T$ (i.e. consists of voters from 3 through $N)$. Moreover, the $\gamma$ 's so obtained are appropriately independent of the extensions used to generate them.

Consider the probabilistic rule $h^{\hat{i}(T)}$ in the general step (Equation 5). Observe that $h^{\hat{i}(T)}$ is well-defined, i.e. all the terms in the summation on the RHS of Equation 5 are known. We will show that it is a strategy-proof RSCF satisfying unanimity. We begin with a preliminary Lemma.

Lemma 13 Suppose $\tau\left(P_{T}^{1}\right)=\tau\left(P_{T}^{2}\right)=a_{T}$. Let $P$ be an $\left(\hat{i}(T), x_{T}\right)$ extension of $\left(P_{T}^{1}, P_{T}^{2}\right)$. Then

$$
\varphi_{\left(b_{T}, x_{-T}\right)}(P)=\sum_{\underline{i}^{g} \in \chi\left(\left(b_{T}, x_{-T}\right), P\right)} \gamma^{g}\left(\underline{i}^{g}\right)
$$

for all $b_{T} \in A_{T}{ }^{6}$

Proof: In view of the definition of an extension, we can assume w.l.o.g $\tau\left(P^{1}\right)=\tau\left(P^{2}\right)=a$. We know from $I H^{*}$ that

$$
\varphi_{\left(b_{T}, x_{-T}\right)}\left(P^{1}, P^{1}, P^{3}, \ldots, P^{N}\right)=\sum_{\underline{i}^{g} \in \chi\left(\left(b_{T}, x_{-T}\right), P\right)} \gamma^{g}\left(\underline{i}^{g}\right)
$$

where $\left(P^{1}, P^{3}, \ldots, P^{N}\right)$ are obtained from the extension $P$. Pick $k \in M-T$ and $c=$ $\left(x_{k}, a_{M-k}\right)$. Assume w.l.o.g. that $\varphi_{c}\left(P^{1}, P^{2}, P^{3}, \ldots, P^{N}\right)>\varphi_{c}\left(P^{1}, P^{1}, P^{3}, \ldots, P^{N}\right)$ (clearly, such a $c$ must exist). Pick $\bar{P}^{2} \in \mathbb{D}^{L}$ such that $a$ and $c$ are the first and second ranked alternatives in $\bar{P}^{2}$ respectively. This is possible by making $k$ the lexicographically worst component. By strategy-proofness

\footnotetext{
${ }^{6}$ We are abusing notation slightly here. In the term $\sum_{\underline{i}^{g} \in \chi\left(\left(b_{T}, x_{-T}\right), P\right)} \gamma^{g}\left(\underline{i}^{g}\right)$ in the expression above, the $P$ in $\chi\left(\left(b_{T}, x_{-T}\right), P\right)$ refers to the $N-1$ profile $\left(P^{1^{\prime}}, P^{3}, \ldots, P^{N}\right)$ where $P^{1^{\prime}}=P^{1}$. We freely use this notation in the rest of the proof - the interpretation should be evident from the context.
} 


$$
\begin{aligned}
\varphi_{a}\left(P^{1}, \bar{P}^{2}, P^{3}, \ldots, P^{N}\right) & =\varphi_{a}\left(P^{1}, P^{2}, P^{3}, \ldots, P^{N}\right) \\
\Rightarrow \varphi_{c}\left(P^{1}, \bar{P}^{2}, P^{3}, \ldots, P^{N}\right) & \geq \varphi_{c}\left(P^{1}, P^{2}, P^{3}, \ldots, P^{N}\right) \\
\Rightarrow \varphi_{c}\left(P^{1}, \bar{P}^{2}, P^{3}, \ldots, P^{N}\right) & >\varphi_{c}\left(P^{1}, P^{1}, P^{3}, \ldots, P^{N}\right) \\
& =\varphi_{c}\left(\bar{P}^{2}, \bar{P}^{2}, P^{3}, \ldots, P^{N}\right)
\end{aligned}
$$

Also, $\varphi_{a}\left(P^{1}, \bar{P}^{2}, P^{3}, \ldots, P^{N}\right)=\varphi_{a}\left(\bar{P}^{2}, \bar{P}^{2}, P^{3}, \ldots, P^{N}\right)$. Hence voter 1 manipulates at $\left(\bar{P}^{2}, \bar{P}^{2}, P^{3}, \ldots, P^{N}\right)$ via $P^{1}$.

Now pick $k, l \in M-T$ and consider $d=\left(d_{k}, d_{l}, a_{M-\{k, l\}}\right)$ such that $\varphi_{d}\left(P^{1}, P^{2}, P^{3}, \ldots, P^{N}\right)>$ $\varphi_{d}\left(P^{1}, P^{1}, P^{3}, \ldots, P^{N}\right)$. We know from the preceding argument that $\varphi_{c}\left(P^{1}, P^{2}, \ldots, P^{N}\right)=$ $\sum_{\underline{i}^{g} \in \chi(c, P)} \gamma^{g}\left(\underline{i}^{g}\right)$ for all $c=\left(c_{j}, a_{M-j}\right)$ and all $j \in M-T$. Now consider $\bar{P}^{2} \in \mathbb{D}^{L}$ where $k$ and $l$ are the lexicographically worst components. Moreover $\bar{P}^{2}$ such that $a=\tau\left(\bar{P}^{2}\right)$ and all alternatives better than $d$ differ from $a$ in exactly one component. We can replicate the earlier argument to show that $\varphi_{d}\left(P^{1}, P^{2}, \ldots, P^{N}\right)=\sum_{\underline{i}^{g} \in \chi(d, P)} \gamma^{g}\left(\underline{i}^{g}\right)$. Moreover, applying the argument repeatedly, we can conclude that $\varphi_{\left(a_{T}, x_{-T}\right)}\left(P^{1}, P^{2}, \ldots, P^{N}\right)=\sum_{\underline{i}^{g} \in \chi\left(\left(a_{T}, x_{-T}\right), P\right)} \gamma^{g}\left(\underline{i}^{g}\right)$. In the same way, we can progressively switch single components in $T$ from $a_{T}$ to some arbitrary $b_{T}$ to show that $\varphi_{\left(b_{T}, x_{-T}\right)}\left(P^{1}, P^{2}, \ldots, P^{N}\right)=\sum_{\underline{i}^{g} \in \chi\left(\left(b_{T}, x_{-T}, P\right)\right.} \gamma^{g}\left(\underline{i}^{g}\right)$.

Our next Lemma establishes that the probabilistic rule $h^{\hat{i}(T)}$ is an RSCF.

Lemma 14 For all $\left(P_{T}^{1}, P_{T}^{2}\right)$,

(i) $\sum_{a_{T} \in A_{T}} h_{a_{T}}^{\hat{i}(T)}\left(P_{T}^{1}, P_{T}^{2}\right)=1$,

(ii) $h_{a_{T}}^{\hat{i}(T)}\left(P_{T}^{1}, P_{T}^{2}\right) \geq 0$ for all $a_{T} \in A_{T}$.

Proof: We begin with $(i)$. Consider first the situation where $\tau\left(P_{T}^{1}\right)=\tau\left(P_{T}^{2}\right)=a_{T}$. In view of Lemma 13, we can assume w.l.o.g. that $P_{T}^{1}=P_{T}^{2}$. Moreover, our extension rule implies that $P^{1}=P^{2}$. We know from Induction Hypothesis* and the definition of $g$ that

$$
\begin{aligned}
\varphi_{\left(a_{T}, x_{-T}\right)}\left(P^{1}, P^{2}, P^{3}, \ldots, P^{N}\right) & =g_{a_{T}}\left(P^{1^{\prime}}, P^{3}, \ldots, P^{N}\right) \\
& =\sum_{\left.\underline{i}^{g} \in \chi\left(a_{T}, x_{-T}\right),\left(P^{1^{\prime}}, P^{3}, \ldots, P^{N}\right)\right)} \gamma^{g}\left(\underline{i}^{g}\right) \\
& =\gamma^{g}(\hat{i}(T))+\sum_{\underline{i \in Z}\left(\left(a_{T}, x_{-T}\right),\left(P^{1^{\prime}}, P^{3}, \ldots, P^{N}\right), T\right)} \gamma(\underline{i})
\end{aligned}
$$

The last step in Equation 8 holds for the following reasons. Since $\tau\left(P_{k}^{1}\right)=\tau\left(P_{k}^{2}\right) \neq x_{k}$ for all $k \in M-T$, it follows that if $\left.\underline{i}^{g} \in \chi\left(a_{T}, x_{-T}\right),\left(P^{1^{\prime}}, P^{3}, \ldots, P^{N}\right)\right), 1^{\prime}$ can appear only 
for components in $T$. Therefore $\chi\left(\left(a_{T}, x_{-T}\right),\left(P^{1^{\prime}}, P^{3}, \ldots, P^{N}\right)\right)$ can be partitioned into two components, one being $\hat{i}(T)$ and the other being $Z\left(\left(a_{T}, x_{-T}\right),\left(P^{1^{\prime}}, P^{3}, \ldots, P^{N}\right)\right)$.

Now using Equation 5, it follows that $h_{a_{T}}^{\hat{i}(T)}\left(P_{T}^{1}, P_{T}^{2}\right)=1$. Consider $b_{T} \neq a_{T}$. Note that $\hat{i}(T) \notin \chi\left(\left(b_{T}, x_{-T}\right),\left(P^{1^{\prime}}, P^{3}, \ldots, P^{N}\right)\right)$. Using Equation 5 once again, we infer that $h_{b_{T}}^{\hat{i}(T)}\left(P_{T}^{1}, P_{T}^{2}\right)=0$.

Consider an arbitrary profile $\left(P_{T}^{1}, P_{T}^{2}\right)$. We can start with the profile $\left(P_{T}^{2}, P_{T}^{2}\right)$ and reach $\left(P_{T}^{1}, P_{T}^{2}\right)$ by switching adjacent "blocks" of alternatives in $P_{T}^{1}$. The total probability on two adjacent blocks is conserved under switching of these blocks by the strategy-proofness of $\varphi$. It follows that the sum of the probabilities of the maximal elements of $P_{T}^{1}$ and $P_{T}^{2}$ in $h^{\hat{i}(T)}$ remains unchanged at the RHS of Equation 8. This implies that the total probability of all alternatives in the domain of $h^{\hat{i}(T)}$ is one.

We now consider $(i i)$. We have already shown in $(i)$ that in the case where $\tau\left(P_{T}^{1}\right)=$ $\tau\left(P_{T}^{2}\right)=a_{T}, h_{a_{T}}^{\hat{i}(T)}\left(P_{T}^{1}, P_{T}^{2}\right)=1$ and $h_{b_{T}}^{\hat{i}(T)}\left(P_{T}^{1}, P_{T}^{2}\right)=0$ for all $b_{T} \neq a_{T}$. Therefore $h_{c_{T}}^{\hat{i}(T)}\left(P_{T}^{1}, P_{T}^{2}\right) \geq$ 0 .

Suppose that $a_{T}=\tau\left(P_{T}^{1}\right) \neq \tau\left(P_{T}^{2}\right)=b_{T}$. Consider an arbitrary alternative $c_{T} \in A_{T}$. Our strategy is to construct another profile by "lowering" $\left(c_{T}, x_{-T}\right)$ as far as possible while making the maximal alternatives of voters 1 and 2 (in the set $T$ ) agree on at least one component in $T$. We can then use the induction step the probability rule $h^{\hat{i}\left(T^{\prime}\right)}$ defined over component set $\left|T^{\prime}\right|<k+1$, is an RSCF to conclude that probability of $c_{T}$ in this profile (under $h^{\hat{i}(T)}$ or $\left(c_{T}, c_{-T}\right)$ under $\left.\varphi\right)$, is non-negative. Now strategy-proofness of $\varphi$ implies that the probability of $\left(c_{T}, x_{-T}\right)$ in the original profile is no lower than its probability in the constructed profile which we have argued to be non-negative.

In the special case where $c_{T}$ differs from $a_{T}$ and $b_{T}$ for all components in $T$, note that in the domain of $h^{\hat{i}(T)}, c_{T}$ can be made last and both voters made to have a common maximal alternative in $A_{T}$ (either $b_{T}$ or $a_{T}$ ).

Suppose that $c_{T}$ agrees with $a_{T}$ for some component $k \in T$. If $c_{k}=b_{k}$, then $a_{k}=b_{k}$ and the non-negativity of $h_{c_{T}}^{\hat{i}(T)}$ follows immediately from the induction step. Assume therefore that $c_{k} \neq b_{k}$. Construct a preference ordering $\bar{P}_{T}^{1}$ for voter 1 such that

(i) $b_{k}$ and $a_{k}$ are the best and worst elements in $A_{k}$ according to $\bar{P}_{k}^{1}$.

(ii) $k$ is the lexicographically worst component in $M$.

(iii) $\bar{P}_{T-k}^{1}=P_{T-k}^{1}$ (this has been defined earlier).

It follows from our construction that $B\left(c_{T}, P_{T}^{1}\right) \subset B\left(c_{T}, \bar{P}_{T}^{1}\right)$ (in the domain of $h^{\hat{i}(T)}$; for $\varphi$, we append $x_{-T}$ to $c_{T}$ everywhere.) By strategy-proofness of $\varphi$, the probability of $\left(c_{T}, x_{-T}\right)$ under $\varphi$ in the profile $\left(P^{1}, P^{2}, \ldots, P^{N}\right)$ is at least as great as the probability of the same alternative in $\left(\bar{P}^{1}, P^{2}, \ldots, P^{N}\right)$. However, we know the induction step on the $|T|$ that the 
probability of the latter $\left(\bar{P}^{1}, P^{2}\right)$ under $h^{\hat{i}(T)}$ is non-negative. This concludes our argument.

Observe that we have already shown that $h^{\hat{i}(T)}$ satisfies unanimity. The strategy-proofness of $h^{\hat{i}(T)}$ follows straightforwardly from its construction and the strategy-proofness of $\varphi$ (we omit the details of the argument). It now follows from Step 1 that $h^{\hat{i}(T)}$ is a generalized random dictatorship. Let the weights associated with this generalized random dictatorships be denoted by $\gamma^{h}(\underline{i}(T))$. Once again $\underline{i}(T)$ is a voter sequence over components in $T$ with every element of the sequence being either voter 1 or 2 . As before let,

$$
\gamma\left(\underline{i}(T), \hat{i}(T)_{-T}\right)=\gamma^{g}(\hat{i}(T)) \cdot \gamma_{\hat{i}(T)}^{h}(\underline{i}(T))
$$

This procedure allows us to obtain the probability weights $\gamma$ for all voter sequences where the cloned voter appears over a set of components of size $k+1$. Note that these probability weights obtained at this Step depend on the extension $x_{T}$. In order to complete Step $B$, we need to show that these weights are, in fact, independent of the $x_{-T}$ chosen. this generalized random dictatorship is appropriately extension-independent.

LEMMA 15 Let $\gamma=\gamma\left(\underline{i}(T), \hat{i}(T)_{-T}\right)$ and $\gamma^{\prime}=\gamma^{\prime}\left(\underline{i}(T), \hat{i}(T)_{-T}\right)$ be the probability weights associated with the extensions $x_{-T}$ and $y_{-T}$ respectively. Then $\gamma=\gamma^{\prime}$.

Proof: We shall follow the arguments in Lemma 12 closely. As before, assume further that $x_{-T}$ and $y_{-T}$ differs over a single component, say $k$. For convenience, let the voter in the $k$ th component of the voter sequence $\left(\underline{i}(T), \hat{i}(T)_{-T}\right)$ be denoted by $s$. Pick a profile $P \in\left[\mathbb{D}^{l}\right]^{N}$ such that

(i) $\tau\left(P_{k}^{s}, A_{k}\right)=x_{k}$.

(ii) The second-ranked alternative in the $k$-th component ranking $P_{k}^{s}$ is $y_{k}$.

(iii) $k$ is the lexicographic worst component in $P^{s}$.

(iv) $\tau\left(P_{j}^{1}, A_{j}\right)=\tau\left(P_{j}^{s}, A_{j}\right)=a_{j} \neq b_{j}=\tau\left(P_{j}^{2}, A_{j}\right)$ for all $j \in T$.

(v) $P$ is a $\left(\hat{i}(T), x_{-T}\right)$ extension of $\left(P_{T}^{1}, P_{T}^{2}\right)$.

Let $\left(a_{T}, x_{k}, d_{M-T-k}\right)$ be the first-ranked alternative in $P^{s}$. By construction, the secondranked alternative in this ordering is $\left(a_{T}, y_{k}, d_{M-T-k}\right)$. Using the definition of a generalized random dictatorship, the total probability on these alternatives in in the profile $P$ under $\varphi$, is given by

$$
\gamma \cdot \gamma^{g}(\hat{i}(T))+\sum_{\underline{i} \in Z\left(\left(a_{T}, x_{k}, d_{M-T-k}\right), P, T\right)} \gamma(\underline{i})+\sum_{\left.\underline{i} \in Z\left(\left(a_{T}, y_{k}, d_{M-T-k}\right)\right), P, T\right)} \gamma(\underline{i}) .
$$


Now suppose $s$ changes her announcement to $\bar{P}^{s}$ where the first and second ranked alternatives are interchanged. This is feasible because $k$ is the lexicographic worst component. If $\left(\bar{P}^{s}, P^{-s}\right)$ is not an extension of $\left(P_{j}^{1}, P_{j}^{2}\right)$, there is nothing to be proved. Suppose, on the other hand that it is an extension. By virtue of earlier arguments, the total probability on the first and second ranked alternatives in $P^{s}$ in the profile $P$ is

$$
\gamma^{\prime} \cdot \gamma^{g}(\hat{i}(T))+\sum_{\underline{i} \in Z\left(\left(a_{T}, x_{k}, d_{M-T-k}\right), P, T\right)} \gamma(\underline{i})+\sum_{\left.\underline{i} \in Z\left(\left(a_{T}, y_{k}, d_{M-T-k}\right)\right), P, T\right)} \gamma(\underline{i}) .
$$

Note that the $\gamma$ terms in the summation terms in the RHS of both expressions 9 and 10 are obtained from the earlier steps with cardinality of $T \leq k$. By the induction hypothesis, these $\gamma^{\prime}$ s are independent of the extensions $x_{-T}$ and $y_{-T}$. This justifies the use of the same $\gamma^{\prime}$ 's in the summation terms in the RHS of expressions 9 and 10. If $\gamma \neq \gamma^{\prime}$, the two expressions are different contradicting the strategy-proofness of $\varphi$.

Our arguments thus far can be summarized as follows. We have constructed probability weights $\gamma(\underline{i})$ for every voter sequence $\underline{i} \in I^{m}$ with the following property:

For any $P \in\left[\mathbb{D}^{L}\right]^{N}$ and $a \in A$ such that there does not exist voters $i, j \in I \backslash\{1,2\}$ and $k \in M$ with $\tau\left(P_{k}^{i}, A_{k}\right)=\tau\left(P_{k}^{j}, A_{k}\right)=a_{k}$, we have

$$
\varphi_{a}(P)=\sum_{\underline{i} \in \chi(a, P)} \gamma(\underline{i})
$$

Equation 11 is obtained by rearranging terms in Equation 5 and using our definition for $\gamma$. In order to complete the proof of the Theorem, we will show that Equation 11 holds for all profiles and alternatives.

Consider a profile $P$ and an alternative $a$ such that there exists exactly one component, say $k$ and exactly two voters $i, j \in I \backslash\{1,2\}$ such that $\tau\left(P_{k}^{i}, A_{k}\right)=\tau\left(P_{k}^{j}, A_{k}\right)=a_{k}$ and $\tau\left(P_{k}^{1}, A_{k}\right), \tau\left(P_{k}^{2}, A_{k}\right) \neq a_{k}$, i.e. for all voters other than 1 and 2 , their maximal alternatives over components $l \neq k$ differs from $a_{l}$. Moreover, all voters than $i$ and $j$ have maximal alternatives in $A_{k}$ different from $a_{k}$. We will show that Equation 11 holds for this $P$.

Let $c_{k} \in A_{k}$ be such that it differs from the maximal alternative in $A_{k}$ for all voters (such an alternative exists because $A_{k}$ has at least three alternatives). Assume w.l.o.g that $k$ is the lexicographically worst component and $b_{k}$ is second-ranked alternative in $A_{k}$ (after $a_{k}$ ) for voter $i$. Now consider an ordering $\bar{P}^{i}$ where the top two alternatives in $P_{k}^{i}$ are switched (this is feasible by assumption). Observe that Equation 5 holds for the profile $\left(\bar{P}^{i}, P^{-i}\right)$ for the alternatives $a$ and $\left(b_{k}, a_{-k}\right)$. Therefore, 


$$
\begin{aligned}
\varphi_{a}\left(\bar{P}^{i}, P^{-i}\right)+\varphi_{\left(b_{k}, a_{-k}\right)}\left(\bar{P}^{i}, P^{-i}\right) & =\sum_{\underline{i} \in \chi\left(a,\left(\bar{P}^{i}, P^{-i}\right)\right)} \gamma(\underline{i})+\sum_{\underline{i} \in \chi\left(\left(b_{k}, a_{-k}\right),\left(\bar{P}^{i}, P^{-i}\right)\right)} \gamma(\underline{i}) \\
& =\sum_{\underline{i} \in \chi(a, P)} \gamma(\underline{i})
\end{aligned}
$$

Note that the second step in 12 follows because $\chi(a, P)=\chi\left(a,\left(\bar{P}^{i}, P^{-i}\right)\right) \cup \chi\left(\left(b_{k}, a_{-k}\right)\left(\bar{P}^{i}, P^{-i}\right)\right)$. We will show that the LHS of Equation 12 in $\varphi_{a}(P)$.

Suppose that the claim above is false, i.e. $\varphi_{\left(b_{k}, a_{-k}\right)}\left(\bar{P}^{i}, P^{-i}\right)>0$. We can assume w.l.o.g that $\left(b_{k}, a_{-k}\right)$ is the worst element in $P^{2}$ (the only restrictions on $P^{2}$ require its maximal elements to be different from those of the others). If voter 2 matches its maximal alternative with that of voter 1 , the probability on $\left(b_{k}, a_{-k}\right)$ can be made to equal zero. This will be a manipulation by voter 2 . Therefore $\varphi_{a}(P)=\sum_{\underline{i \in \chi(a, P)}} \gamma(\underline{i})$ as required.

We can apply this argument repeatedly to show that Equation 11 holds for any profile $P$ and any alternative $a$. This completes the proof of the Theorem.

We have proved our result for lexicographically separable preferences. An important open question is whether the result generalizes to separable supersets of this domain.

\section{Conclusion}

We have generalized the random dictatorship result of Gibbard (1977) to a multi-dimensional setting where voter preferences are lexicographically separable. In particular we have shown that strategy-proof random social choice functions satisfying unanimity are generalized random dictatorships. These are induced by a fixed probability distribution on voter sequences of length equal to the number of components. Although the joint distribution on outcomes is not the product of strategy-proof component random social functions, we have shown that the marginal probability distribution on each component at a preference profile depends only on component preferences. Moreover the marginal random social choice functions are in fact, strategy-proof and therefore random dictatorships. An important question for future research is whether the decomposability of the marginal random social choice functions holds more generally, for instance, for "rich domains" as defined in LeBreton and Sen (1999).

\section{REFERENCES}

Barberì, S., F. Gul, And E. Stachetti (1993): "Generalized Median Voter Schemes and Committees," Journal of Economic Theory, 61, 262-289.

BarberÀ, S., J. J. Massó, And A. Neme (1997): "Voting under Constraints," Journal of Economic Theory, 76, 298-321. 
(2005): "Voting by Committees under Constraints," Journal of Economic Theory, $122,185-205$.

Barberì, S. And H. Sonnenschein (1978): "Strategy-proof Voting Schemes with Continuous Preferences," Journal of Economic Theory, 18, 244-254.

BarberÀ, S., H. Sonnenschein, And L. Zhou (1991): "Voting by Committees," Econometrica, 59, 595-609.

Bogomolnaia, A. And H. Moulin (2001): "A New Solution to the Random Assignment Problem," Journal of Economic Theory, 100, 295-328.

- (2004): "Random Matching under Dichotomous Preferences," Econometrica, 72, $2957-279$.

Bogomolnaia, A., H. Moulin, And R. Stong (2005): "Collective choice under dichotomous preferences," Journal of Economic Theory, 122, 165-184.

GibBard, A. (1973): "The Manipulation of Voting Schemes: A General Result," Econometrica, 41, 587-601.

(1977): "Manipulation of Voting Schemes that Mix Voting With Chance," Econometrica, 45, 665-681.

LeBreton, M. And A. Sen (1999): "Separable Preferences, Strategy-proofness and Decomposability," Econometrica, 67, 605-628.

Moulin, H. And R. Stong (2002): "Fair Queueing and other Probabilistic Allocation Methods," Mathematics of Operations Research, 27, 1-31.

Myerson, R. (1981): "Optimal Auction Design," Mathematics of Operations Research, 6, $1767-1797$.

Satterthwaite, M. (1975): "Strategy-proofness and Arrow's Conditions: Existence and Correspondence Theorems for Voting Procedures and Social Welfare Functions," Journal of Economic Theory, 10, 187-217.

SEn, A. (2011): "The Gibbard Random Dictatorship Theorem: A Generalization and a New Proof," Spanish Economic Review (SERIEs), 515-527.

Sprumont, Y. (1995): "Strategy-proof Collective Choice in Economic and Political Environments," Canadian Economic Review, 28, 68-107.

Svensson, L.-G. And Torstensson (2008): "Strategy-proof Allocation of Multiple Public Goods," Social Choice and Welfare, 30, 181-196. 